\title{
Gravitational cubic-in-spin interaction at the next-to-leading post-Newtonian order
}

\author{
Michèle Levi, ${ }^{a, b}$ Stavros Mougiakakos ${ }^{b}$ and Mariana Vieira ${ }^{a}$ \\ ${ }^{a}$ Niels Bohr International Academy, Niels Bohr Institute, University of Copenhagen, \\ Blegdamsvej 17, 2100 Copenhagen, Denmark \\ ${ }^{b}$ Institut de Physique Théorique, CEA \& CNRS, Université Paris-Saclay, \\ 91191 Gif-sur-Yvette, France \\ E-mail: michelelevi@nbi.ku.dk, stavros.mougiakakos@ipht.fr, \\ dcp182@alumni.ku.dk
}

ABSTRACT: In this work we derive for the first time the complete gravitational cubic-inspin effective action at the next-to-leading order in the post-Newtonian (PN) expansion for the interaction of generic compact binaries via the effective field theory for gravitating spinning objects, which we extend in this work. This sector, which enters at the fourth and a half $\mathrm{PN}$ (4.5PN) order for rapidly-rotating compact objects, completes finite-size effects up to this PN order, and is the first sector completed beyond the current state of the art for generic compact binary dynamics at the $4 \mathrm{PN}$ order. At this order in spins with gravitational nonlinearities we have to take into account additional terms, which arise from a new type of worldline couplings, due to the fact that at this order the Tulczyjew gauge for the rotational degrees of freedom, which involves the linear momentum, can no longer be approximated only in terms of the four-velocity. One of the main motivations for us to tackle this sector is also to see what happens when we go to a sector, which corresponds to the gravitational Compton scattering with quantum spins larger than one, and maybe possibly also get an insight on the inability to uniquely fix its amplitude from factorization when spins larger than two are involved. A general observation that we can clearly make already is that even-parity sectors in the order of the spin are easier to handle than odd ones. In the quantum context this corresponds to the greater ease of dealing with bosons compared to fermions.

KEywords: Classical Theories of Gravity, Effective Field Theories, Black Holes, Scattering Amplitudes

ArXiv ePrint: 1912.06276 


\section{Contents}

1 Introduction 1

2 The EFT of gravitating spinning objects 4

3 The essential computation $\quad 6$

3.1 One-graviton exchange $\quad 7$

$\begin{array}{lll}3.2 & \text { Two-graviton exchange } & 12\end{array}$

$\begin{array}{lll}3.3 & \text { Cubic self-interaction } & 14\end{array}$

4 New features from spin dependence of linear momentum 16

5 The gravitational cubic-in-spin action at the next-to-leading order $\quad 19$

6 Conclusions 24

\section{Introduction}

Since the first detection of gravitational waves (GWs) from a binary black hole coalescence was announced in 2016 [1], it has become increasingly pressing to provide high-precision theoretical predictions for the modeling of GW templates. The latter rely on implementing analytical results obtained within the post-Newtonian (PN) approximation of classical Gravity [2] via the Effective-One-Body approach [3]. In particular in recent years we have made a remarkable progress in pushing the precision frontier for the orbital dynamics of compact binaries, i.e. whose components are generic compact objects, such as black holes or neutron stars. The complete state of the art to date for the orbital dynamics of a generic compact binary is shown in table 1 .

As a measure for the loop computational scale we show in table 1 the number of $n$ loop graphs that enter at the $\mathrm{N}^{n} \mathrm{LO}$ in $l$ powers of the spin, i.e. up to the $l$ th spin-induced multipole moment, in the sectors approached to date. The count is based on computations carried out with the effective field theory (EFT) of PN Gravity [4], which use the KaluzaKlein decomposition of the field from [5], that has considerably facilitated high-precision computations within the EFT approach [5-20]. As can be seen the current complete state of the art is at the $4 \mathrm{PN}$ order, whereas the next-to-leading order (NLO) cubic-inspin sector that enters at the $4.5 \mathrm{PN}$ order for maximally-rotating objects is evaluated in this paper. All of the sectors at the current state of the art (but the top right entry at the $4 \mathrm{PN}$ order for the non-rotating case) are available in the public EFTofPNG code at https://github.com/miche-levi/pncbc-eftofpng [21].

Let us stress that in order to attain a certain level of PN accuracy, the various sectors should be tackled across the diagonals of table 1, rather than along the axes, namely 


\begin{tabular}{|l|r|r|r|r|r|}
\hline \multicolumn{1}{|c|}{$\boldsymbol{n}$} & $\left(\mathrm{N}^{\mathbf{0}}\right) \mathrm{LO}$ & $\mathrm{N}^{(\mathbf{1})} \mathrm{LO}$ & $\mathrm{N}^{2} \mathrm{LO}$ & $\mathrm{N}^{\mathbf{3}} \mathrm{LO}$ & $\mathrm{N}^{4} \mathrm{LO}$ \\
\hline $\mathrm{S}^{\mathbf{0}}$ & 1 & 0 & 3 & 0 & 25 \\
\hline $\mathrm{S}^{\mathbf{1}}$ & 2 & 7 & 32 & 174 & \\
\hline $\mathrm{S}^{\mathbf{2}}$ & 2 & 2 & 18 & 52 & \\
\hline $\mathrm{S}^{\mathbf{3}}$ & 4 & 24 & & & \\
\hline $\mathrm{S}^{\mathbf{4}}$ & 3 & 5 & & & \\
\hline
\end{tabular}

Table 1. The complete state of the art of PN gravity theory for the orbital dynamics of generic compact binaries. Each PN correction enters at the order $n+l+\operatorname{Parity}(l) / 2$, where the parity is 0 or 1 for even or odd $l$, respectively. We elaborate on the meaning of the numerical entries and the gray area in the text.

progress must be made by going in parallel to higher loops and to higher orders of the spin. In general, the former involves more computational challenges of loop technology and tackling associated divergences, whereas the latter necessitates an improvement of the fundamental understanding of spin in gravity, and tackling finite-size effects with spin [22]. The latter enter first at the $2 \mathrm{PN}$ order [23] from the LO spin-induced quadrupole. Within the EFT approach, whose extension to the spinning case was first approached in [24], finite-size effects include as additional parameters the Wilson coefficients, that correspond, e.g., to the multipole deformations of the object due to its spin, as in [25] for the spininduced quadrupole.

With a considerable time gap from the LO result, the NLO spin-squared interaction was treated in a series of works [12, 26-29], where in [12] it was derived within the formulation of the EFT for gravitating spinning objects, that provided the leading non-minimal couplings to all orders in spin. The LO cubic- and quartic-in-spin interactions were first tackled in $[28,30]$ for black holes. In [11], based on the formulation presented in [12], these were derived for generic compact objects, where also the quartic-in-spin interaction was completed. Only specific pieces of the latter were recovered in [31] via S-matrix combined with EFT techniques, whereas [32], which treated only cubic-in-spin effects, also provided the LO effects in the energy flux. Following the work in $[11,12]$, the case of black holes was then also completed for the LO sectors to all orders in spin [33]. Finally, the NNLO spin-squared interaction was derived in [14]. Notably the latter results together with the complete quartic-in-spin results for generic compact objects in [11], both at the 4PN order, were derived exclusively within the EFT formulation of spinning gravitating objects [12]. Building on the latter, recent works further pushed at the 4.5PN [18] and 5PN [19, 20] orders for maximally-spinning objects.

Recently, there has also been a surge of interest in harnessing modern advances in scattering amplitudes to the problem of a coalescence of a compact binary. Notably, a new implementation for the non-rotating case to the derivation of classical potentials was carried out in $[34,35]$. Further, based on a new spinor-helicity formalism introduced in [36] for massive particles of any spin, new approaches to the computation of spin effects of black holes in the classical potential were put forward in $[37,38]$ and then in $[39,40]$. 


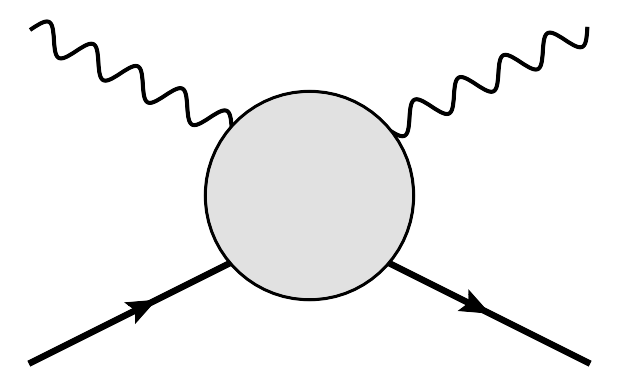

Figure 1. The gravitational Compton scattering relevant as of the one-loop level. The gravitational Compton amplitude involves two massive spinning particles and two gravitons, where factorization constraints do not uniquely determine the amplitude for $s>2[36]$.

In these approaches classical effects with spin to the $l$ th order correspond to scattering amplitudes involving a quantum spin of $s=l / 2$. In particular as of the one-loop level the gravitational Compton amplitude in figure 1 is required, where factorization constraints can not uniquely determine the amplitude for $s>2$ [36]. The gray area in table 1 then corresponds in the quantum context to where the gravitational Compton scattering with a spin $s>1$ is required.

Notably, the gray area in table 1 also corresponds to, as was already pointed out in [12], where we can no longer take the linear momentum $p_{\mu}$, with which the generic formulation in [12] was made, to be its leading approximation given by $m \frac{u^{\mu}}{\sqrt{u^{2}}}$, as in all previously tackled spin sectors, but we have to take into account corrections to the linear momentum from the non-minimal coupling part of the spinning particle action. Can we then get a well-defined result? Can we get an insight from examining this new feature at the classical level on the non-uniqueness of the graviton Compton amplitude with $s>2$ ?

This work builds on the formalism of the EFT for gravitating spinning objects introduced in [12] and the implementation on [11], to compute the cubic-in-spin interaction at the NLO, that enters at the 4.5PN order for maximally-rotating compact objects, pushing the current state of the art of PN theory in general and with spins in particular [41], and that is the leading sector in the intriguing gray area of table 1 . We compute the complete sector, taking into account all interactions that include all possible spin multipoles terms up to and including the octupole. Thus beyond pushing the state of the art in PN theory, there are two conceptual objectives that we get to address in this work: 1. To learn how the spin dependence of the linear momentum affects the results in the interaction; 2 . To see whether this new feature is related with the non-uniqueness of the gravitational Compton amplitude of higher-spin states, or get any possible insight on this non-uniqueness.

The paper is organized as follows. In section 2 we go over the formulation from [12], and the necessary ingredients to evaluate the more familiar part of the sector. In section 3 we present the essential computation, where the linear momentum assumes its leading approximation in terms of the four-velocity, as in all past evaluations of spin sectors. In section 4 we find the new contributions arising from the spin-dependent correction to the linear momentum, which matters as of this sector, and gives rise to a new type of worldlinegraviton coupling. In section 5 we assemble the final action of this sector, and finally we conclude in section 6 with some observations and questions. 


\section{The EFT of gravitating spinning objects}

Let us consider the ingredients of the theory that are required in order to carry out the evaluation of this sector, that contains spins up to cubic order along with first gravitational nonlinearities. This evaluation will build on the EFT of gravitating spinning objects formulated in [12], and its implementation from LO up to the state of the art at the 4PN order in [11-14, 41]. We will also use here the Kaluza-Klein decomposition of the field [5, 42], which was adopted in all high-order PN computations both with and without spins for its facilitating virtues [22], and follow conventions consistent with the abovementioned works. Further, we keep similar gauge choices, notational and pictorial conventions as presented in $[12]$.

The effective action we start from is that of a two-particle system [22], with each of the particles described by the effective point-particle action of a spinning particle, that was provided in [12]. This effective action contains a purely gravitational piece, from which the propagators and self-interacting vertices are derived. The Feynman rules for the propagator and the time insertions on the propagators are given, e.g. in eqs. (5)-(10) of [10], and for the cubic gravitational vertices in eqs. (2.10)-(2.13), and (2.15) of [13]. Further, for each of the two particles, the worldline action of a spinning particle is considered from [12], where its non-minimal coupling spin-induced part was constructed to all orders in spin, and then gauge freedom of the rotational DOFs is incorporated into the action.

We recall that this action has the form

$$
S_{\mathrm{pp}}(\sigma)=\int d \sigma\left[-m \sqrt{u^{2}}-\frac{1}{2} \hat{S}_{\mu \nu} \hat{\Omega}^{\mu \nu}-\frac{\hat{S}^{\mu \nu} p_{\nu}}{p^{2}} \frac{D p_{\mu}}{D \sigma}+L_{\mathrm{SI}}\right],
$$

given in terms of the four-velocity $u^{\mu}$, the linear momentum $p_{\mu}$, and the rotational DOFs in a generic gauge, denoted with a hat, e.g. $\hat{S}_{\mu \nu}$, and the label "SI" stands for the spin-induced non-minimal coupling part of the action. For the sector evaluated here the latter part will consist of its two leading terms given by

$$
L_{\mathrm{SI}}=-\frac{C_{E S^{2}}}{2 m} \frac{E_{\mu \nu}}{\sqrt{u^{2}}} S^{\mu} S^{\nu}-\frac{C_{B S^{3}}}{6 m^{2}} D_{\lambda} \frac{B_{\mu \nu}}{\sqrt{u^{2}}} S^{\mu} S^{\nu} S^{\lambda},
$$

in which we use the definite-parity curvature components defined as

$$
\begin{aligned}
E_{\mu \nu} & \equiv R_{\mu \alpha \nu \beta} u^{\alpha} u^{\beta}, \\
B_{\mu \nu} & \equiv \frac{1}{2} \epsilon_{\alpha \beta \gamma \mu} R_{\delta \nu}^{\alpha \beta} u^{\gamma} u^{\delta},
\end{aligned}
$$

for the electric and magnetic components of even and odd parity, respectively. Notice also that here we need to use the Levi-Civita tensor density in curved spacetime, $\epsilon_{\alpha \beta \gamma \lambda}=$ $\sqrt{-g} e_{\alpha \beta \gamma \lambda}$, in which $g$ is the determinant of $g_{\mu \nu}$, and $e_{\alpha \beta \gamma \lambda}$ is the totally antisymmetric Levi-Civita symbol with $e_{0123}=+1$. We note also that we use here a classical version of the Pauli-Lubanski pseudovector, $S^{\mu}$, as first defined in [11]

$$
S_{\mu}=\frac{1}{2} \epsilon_{\alpha \beta \gamma \mu} S^{\alpha \beta} \frac{p^{\gamma}}{p}
$$


which is with a reverse sign with respect to the definition in [12], that was applied up to the quadratic-in-spin order, where this sign choice does not matter. The spin tensor that is used in eq. (2.5) is related to the spin in the generic gauge $\hat{S}_{\mu \nu}$ via the projection of the latter onto the spatial hypersurface of the rest frame according to eq. (3.29) in [12].

We recall that in eq. (2.1) there is an extra term, which appears in the action from the restoration of gauge freedom of the rotational DOFs. This term, which is essentially the Thomas precession, as discussed in detail in [12] (and recovered recently as "Hilbert space matching" in $[40,43])$, contributes to all orders in the spin as of the LO spin-orbit sector, and in particular also to finite-size spin effects, though it does not encode any UV physics, but rather in the context of an effective action, just accounts for the fact that a relativistic gravitating object has an extended measure.

Since we compute here the complete NLO cubic-in-spin sector our graphs will contain all multipoles in the presence of spin up to the spin-induced octupole, i.e. also including the mass, spin and spin-induced quadrupole. For this reason we need to use Feynman rules of worldline-graviton coupling to NLO for all of these multipoles, where in this work we need to derive further new rules for the octupole couplings. The Feynman rules required for the mass couplings are given in eqs. (64), (67), (68), (79), (81), (93), (95) of [8]. Next, we approach the Feynman rules linear in spin, noting that first we have kinematic contributions as noted in eq. (5.28) of [12], that are linear in the spin but have no field coupling, which we will take into account in section 5 . The Feynman rules required for the linear-in-spin couplings are given in eqs. (5.29)-(5.31) of [12], and eqs. (2.31)-(2.34) of [13]. For the spin quadrupole couplings the rules are given in eqs. (2.18)-(2.24) of [14], and for the LO spin octupole couplings they are found in eqs. (2.19), (2.20) of [11].

As we noted in addition to the abovementioned Feynman rules, further rules are required here for the spin-induced octupole worldline-graviton coupling. The two Feynman rules of the scalar and vector components of the KK decomposition, which appeared already at LO in [11] should be extended to a higher PN order, and further we will have new rules that enter for the one-graviton coupling of the tensor component of the KK fields, and a couple of two-graviton couplings, involving again the scalar and the vector components of the KK fields.

The extended rules for the one-graviton couplings are then given as follows:

$$
\begin{aligned}
& \prod_{---}=\int d t\left[\frac { C _ { \mathrm { BS } ^ { 3 } } } { 1 2 m ^ { 2 } } S _ { i } S _ { j } \epsilon _ { k l m } \left[A_{k, i j l}\left(S_{m}\left(1+\frac{1}{2} v^{2}\right)-\frac{1}{2} v^{m} S_{n} v^{n}\right)\right.\right. \\
& \left.\left.+S_{m}\left(v^{l} v^{n}\left(A_{i, n j k}-A_{n, i j k}\right)+v^{l}\left(\partial_{t} A_{k, i j}+\partial_{t} A_{i, j k}\right)+v^{i} \partial_{t} A_{k, j l}\right)\right]\right], \\
& \varsubsetneqq=\int d t\left[\frac{C_{\mathrm{BS}^{3}}}{3 m^{2}} S_{i} S_{j} \epsilon_{k l m} S_{m} v^{l}\left(\phi_{, i j k}\left(1+\frac{v^{2}}{2}\right)+v^{i} \partial_{t} \phi_{, j k}\right)\right],
\end{aligned}
$$

in which the rectangular boxes represent the spin-induced octupole. 
The new Feynman rules required here are given as follows:

$$
\varsubsetneqq=\int d t\left[\frac{C_{\mathrm{BS}^{3}}}{12 m^{2}} S_{i} S_{j} \epsilon_{k l m} S_{m} \partial_{i} \partial_{l}\left(\left(\partial_{j} \sigma_{k n}-\partial_{n} \sigma_{j k}\right) v^{n}-\partial_{t} \sigma_{j k}\right)\right],
$$

for the one-graviton coupling, whereas for the two-graviton couplings we get:

$$
\begin{gathered}
\left.\left.+4 \phi_{, i j} A_{k, l}+4 \phi_{, j k}\left(A_{i, l}-A_{l, i}\right)+\delta_{i j} \phi_{, n} A_{l, k n}\right)\right], \\
=\int d t\left[\frac { C _ { \mathrm { BS } ^ { 3 } } } { 1 2 m ^ { 2 } } S _ { i } S _ { j } \epsilon _ { k l m } S _ { m } \left(6 \phi A_{k, i j l}+9 \phi_{, i} A_{k, j l}+3 \phi_{, k} \partial_{j}\left(A_{i, l}-A_{l, i}\right)\right.\right. \\
=\int \frac{C_{\mathrm{BS}^{3}}}{3 m^{2}} S_{i} S_{j} \epsilon_{k l m} S_{m}\left[v^{l}\left(2 \phi_{, i j k} \phi+3 \phi_{, i j} \phi_{, k}+5 \phi_{, i} \phi_{, j k}-\delta_{i j} \phi_{, n} \phi_{, k n}\right)\right. \\
\left.\left.+v^{i} \phi_{, l j} \phi_{, k}\right]\right] .
\end{gathered}
$$

We note that in these rules the spin is already fixed to the canonical gauge in the local frame, and all indices are Euclidean. Notice the complexity of these couplings with respect to other worldline couplings at the NLO level, and notice also the dominant role that the gravitomagnetic vector plays in the coupling to the odd-parity octupole, similar to the situation in the coupling to the spin dipole. This is the first sector which necessitates to actually take the curved Levi-Civita tensor and the covariant derivative into account.

For this sector there is no need to extend the non-minimal coupling part of the spinning particle action and add higher dimensional operators beyond what was provided in [12], but we need to pay special attention to the new feature that differentiates this specific sector from all the spin sectors which were tackled in the past. In this sector it is no longer sufficient to use the leading approximation for the linear momentum $p_{\mu}$ in terms of the four-velocity $u_{\nu}$ all throughout, rather one has to take into account the subleading term in the linear momentum, which is linear in Riemann and quadratic in the spin, and becomes relevant first once we get to the level that is cubic in the spins and non-linear in gravity, i.e. at this sector, as was already explicitly noted in [12]. We will address in detail the particular contributions coming from this new feature in section 4 below, after we have done in the following section the essential computation, which requires only the leading approximation to the linear momentum that is independent of spin, similar to what was considered in all past PN computations in spin sectors.

\section{The essential computation}

In this section we carry out the perturbative expansion of the effective action in terms of Feynman graphs, and provide the value of each diagram, while using the leading approximation of the linear momentum. At the NLO level, i.e. up to the $G^{2}$ order, all of the three relevant topologies are realized with spins, even when the beneficial KK decomposition of 
the field is used, as discussed in $[6,8,12,22]$. As shown in figures $2-4$ below (drawn using Jaxodraw [44, 45] based on [46]) there is a total of $49=10+15+24$ graphs making up this part of the sector, distributed among the relevant topologies of one- and two-graviton exchanges and cubic self-interaction, respectively. As shown in table 1 about half of the total graphs require a one-loop evaluation (the highest loop in this sector). We note that as we go into the nonlinear part of the sector, the options for the make-up of the interaction become more intricate.

At the one-graviton exchange level we only have two kinds of interaction contributing, similar to the LO in [11], namely either an octupole-monopole or a quadrupole-dipole interaction. As noted in [11] there are nice analogies among these interactions according to the parity of the multipole moments involved. Following these analogies the relevant graphs of one-graviton exchange are easily constructed. Yet, once we proceed to the level nonlinear in the gravitons further types of interactions emerge. In particular, there are also interactions involving various multipoles on two different points of the worldline, which add up to interactions that are cubic in the spin, such as a spin and a spin-induced quadrupole or two spin dipoles, on the same worldline, which can already be seen as of the NLO spin-squared sector $[12,14]$.

We note that all the graphs in this sector should be included together with their mirror images, i.e. with the worldline labels $1 \leftrightarrow 2$ exchanged. For more specific details on the generation of the Feynman graphs, and their evaluation, including the conventions and notations used here, we refer the reader to [22] and references therein. We note that the generation and the evaluation of the graphs was crosschecked using the publicly-available EFTofPNG code [21].

\section{$3.1 \quad$ One-graviton exchange}

As can be seen in figure 2 we have 10 graphs of one-graviton exchange in this sector, the majority of which already involve time derivatives to be applied. Consistent with former works by one of the authors we keep all of the higher order time derivative terms that emerge in the evaluations of the graphs, and they will be treated properly via redefinitions of the position and the rotational variables as shown in [47]. Notice that we have here the 4 graphs that appeared at the LO with the quadratic time insertions on the propagators at graphs 1(a7)-(a10), and a new octupole coupling involving the KK tensor field at graph 1(a3).

The graphs in figure 2 are evaluated as follows:

Fig. $2(\mathrm{a} 1)=-C_{1\left(B S^{3}\right)} \frac{G}{r^{4}} \frac{m_{2}}{m_{1}^{2}}\left[\vec{S}_{1} \cdot \vec{v}_{1} \times \vec{v}_{2}\left(2 \vec{S}_{1} \cdot \vec{v}_{2} \vec{S}_{1} \cdot \vec{n}\right.\right.$

$$
\begin{aligned}
& \left.+\vec{v}_{2} \cdot \vec{n}\left(S_{1}^{2}-5\left(S_{1} \cdot \vec{n}\right)^{2}\right)-\vec{S}_{1} \cdot \vec{v}_{1} \vec{S}_{1} \cdot \vec{n}\right) \\
& +\vec{S}_{1} \cdot \vec{v}_{1} \times \vec{n}\left(\left(S_{1}^{2}-5\left(\vec{S}_{1} \cdot \vec{n}\right)^{2}\right) \vec{v}_{1} \cdot \vec{v}_{2}+\vec{S}_{1} \cdot \vec{v}_{2}\left(\vec{S}_{1} \cdot \vec{v}_{2}-5 \vec{S}_{1} \cdot \vec{n} \vec{v}_{2} \cdot \vec{n}\right)\right. \\
& \left.-\vec{S}_{1} \cdot \vec{v}_{2}\left(\vec{S}_{1} \cdot \vec{v}_{1}-5 \vec{S}_{1} \cdot \vec{n} \vec{v}_{1} \cdot \vec{n}\right)\right)+\vec{S}_{1} \cdot \vec{v}_{2} \times \vec{n}\left(\frac{1}{2} S_{1}^{2}\left(v_{1}^{2}+v_{2}^{2}\right)\right. \\
& \left.-\vec{S}_{1} \cdot \vec{v}_{1}\left(\vec{S}_{1} \cdot \vec{v}_{2}-5 \vec{S}_{1} \cdot \vec{n} \vec{v}_{2} \cdot \vec{n}\right)-\frac{5}{2}\left(\vec{S}_{1} \cdot \vec{n}\right)^{2}\left(v_{1}^{2}+v_{2}^{2}\right)\right)
\end{aligned}
$$




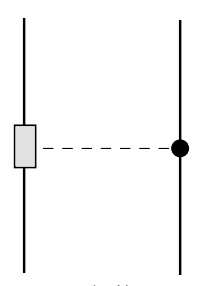

(a1)

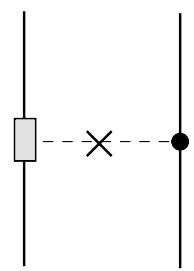

(a7)

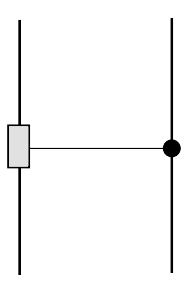

(a2)

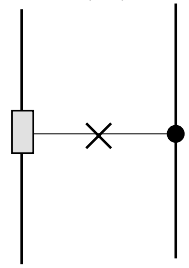

(a8)

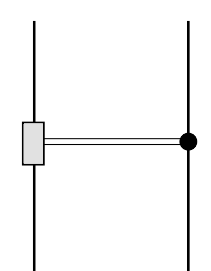

(a3)

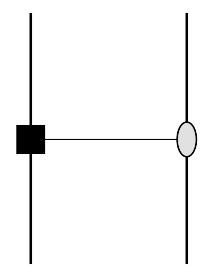

(a4)

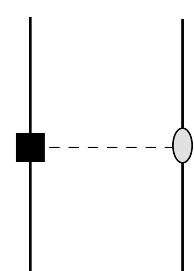

(a5)

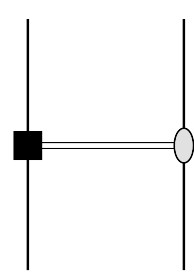

(a6)

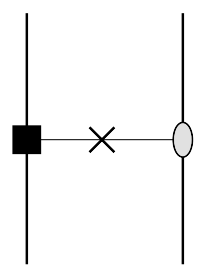

(a9)

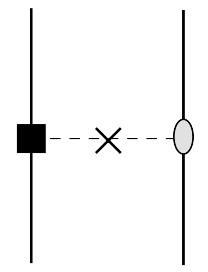

(a10)

Figure 2. The Feynman graphs of one-graviton exchange, which contribute to the NLO cubic-inspin interaction at the $4.5 \mathrm{PN}$ order for maximally rotating compact objects. The graphs should be included together with their mirror images, i.e. with the worldline labels $1 \leftrightarrow 2$ exchanged. At the linear level of one-graviton exchange we only have two kinds of interactions contributing, similar to the LO in [11], namely either a quadrupole-dipole or an octupole-monopole interaction. As noted in [11] there are nice analogies among these interactions according to the parity of the multipole moments involved. Following these analogies the relevant graphs here are easily constructed. Notice that we have here the four graphs that appeared at the $\mathrm{LO}$ with the quadratic time insertions on the propagators at graphs (a7)-(a10), and a new octupole coupling involving the KK tensor field at graph (a3).

$$
\begin{aligned}
& \left.-\frac{1}{2} \vec{v}_{1} \cdot \vec{v}_{2} \times \vec{n} \vec{S}_{1} \cdot \vec{v}_{1}\left(S_{1}^{2}-5\left(\vec{S}_{1} \cdot \vec{n}\right)^{2}\right)\right] \\
& -\frac{1}{3} C_{1\left(B S^{3}\right)} \frac{G}{r^{3}} \frac{m_{2}}{m_{1}^{2}}\left[\vec{S}_{1} \cdot \vec{v}_{1} \times \vec{a}_{2}\left(S_{1}^{2}-3\left(\vec{S}_{1} \cdot \vec{n}\right)^{2}\right)-3 \vec{S}_{1} \cdot \vec{v}_{1} \times \vec{n} \vec{S}_{1} \cdot \vec{a}_{2} \vec{S}_{1} \cdot \vec{n}\right. \\
& \left.+3 \vec{S}_{1} \cdot \vec{a}_{2} \times \vec{n} \vec{S}_{1} \cdot \vec{v}_{1} \vec{S}_{1} \cdot \vec{n}\right]
\end{aligned}
$$

Fig. $2(\mathrm{a} 2)=\frac{1}{2} C_{1\left(B S^{3}\right)} \frac{G}{r^{4}} \frac{m_{2}}{m_{1}^{2}}\left[\vec{S}_{1} \cdot \vec{v}_{1} \times \vec{n}\left(S_{1}^{2}\left(v_{1}^{2}+3 v_{2}^{2}\right)-2 \vec{S}_{1} \cdot \vec{n}\left(\vec{S}_{1} \cdot \vec{v}_{2}-5 \vec{S}_{1} \cdot \vec{n} \vec{v}_{2} \cdot \vec{n}\right)\right.\right.$

$$
\left.\left.-5\left(\vec{S}_{1} \cdot \vec{n}\right)^{2}\left(v_{1}^{2}+3 v_{2}^{2}\right)\right)-2 \vec{S}_{1} \cdot \vec{v}_{1} \times \vec{v}_{2} \vec{S}_{1} \cdot \vec{v}_{1} \vec{S}_{1} \cdot \vec{n}\right],
$$

Fig. $2(\mathrm{a} 3)=-C_{1\left(B S^{3}\right)} \frac{G}{r^{4}} \frac{m_{2}}{m_{1}^{2}}\left[\left(\vec{S}_{1} \cdot \vec{v}_{1} \times \vec{n} v_{2}^{2}-\vec{S}_{1} \cdot \vec{v}_{2} \times \vec{n} \vec{v}_{1} \cdot \vec{v}_{2}\right)\left(S_{1}^{2}-5\left(\vec{S}_{1} \cdot \vec{n}\right)^{2}\right)\right]$

$$
\begin{aligned}
& +C_{1\left(B S^{3}\right)} \frac{G}{r^{3}} \frac{m_{2}}{m_{1}^{2}}\left[\vec{S}_{1} \cdot \vec{v}_{2} \times \vec{n}\left(\vec{S}_{1} \cdot \vec{v}_{2} \dot{\vec{S}}_{1} \cdot \vec{n}+\dot{\vec{S}}_{1} \cdot \vec{v}_{2} \vec{S}_{1} \cdot \vec{n}\right)\right. \\
& \left.+\dot{\vec{S}}_{1} \cdot \vec{v}_{2} \times \vec{n} \vec{S}_{1} \cdot \vec{v}_{2} \vec{S}_{1} \cdot \vec{n}\right]
\end{aligned}
$$

Fig. $2(\mathrm{a} 4)=\frac{3}{2} C_{1\left(E S^{2}\right)} \frac{G}{r^{4}} \frac{1}{m_{1}}\left[2 \vec{S}_{1} \cdot \vec{S}_{2} \times \vec{v}_{2}\left(\vec{S}_{1} \cdot \vec{v}_{1} \vec{v}_{1} \cdot \vec{n}-\vec{S}_{1} \cdot \vec{n}\left(3 v_{1}^{2}+v_{2}^{2}\right)\right)\right.$

$$
\begin{aligned}
& +2 \vec{S}_{2} \cdot \vec{v}_{1} \times \vec{v}_{2}\left(2 S_{1}^{2} \vec{v}_{1} \cdot \vec{n}-\vec{S}_{1} \cdot \vec{v}_{1} \vec{S}_{1} \cdot \vec{n}\right) \\
& -\vec{S}_{2} \cdot \vec{v}_{2} \times \vec{n}\left(S_{1}^{2}\left(5 v_{1}^{2}+v_{2}^{2}-10\left(\vec{v}_{1} \cdot \vec{n}\right)^{2}\right)-2 \vec{S}_{1} \cdot \vec{v}_{1}\left(\vec{S}_{1} \cdot \vec{v}_{1}-5 \vec{S}_{1} \cdot \vec{n} \vec{v}_{1} \cdot \vec{n}\right)\right.
\end{aligned}
$$




$$
\begin{aligned}
& \left.\left.-5\left(\vec{S}_{1} \cdot \vec{n}\right)^{2}\left(3 v_{1}^{2}+v_{2}^{2}\right)\right)\right] \\
& +C_{1\left(E S^{2}\right)} \frac{G}{r^{3}} \frac{1}{m_{1}}\left[2 \vec{S}_{1} \cdot \vec{S}_{2} \times \vec{v}_{2}\left(\vec{S}_{1} \cdot \vec{a}_{1}+\dot{\vec{S}}_{1} \cdot \vec{v}_{1}\right)+2 \dot{\vec{S}}_{1} \cdot \vec{S}_{2} \times \vec{v}_{2} \vec{S}_{1} \cdot \vec{v}_{1}\right. \\
& +4 \vec{S}_{2} \cdot \vec{v}_{1} \times \vec{v}_{2} \overrightarrow{\vec{S}}_{1} \cdot \vec{S}_{1}+2 \vec{S}_{2} \cdot \vec{v}_{2} \times \vec{a}_{1} S_{1}^{2}+\vec{S}_{2} \cdot \vec{v}_{2} \times \vec{a}_{2}\left(S_{1}^{2}-3\left(\vec{S}_{1} \cdot \vec{n}\right)^{2}\right) \\
& -6 \vec{S}_{2} \cdot \vec{v}_{2} \times \vec{n}\left(S_{1}^{2} \vec{a}_{1} \cdot \vec{n}-2 \dot{\vec{S}}_{1} \cdot \vec{S}_{1} \vec{v}_{1} \cdot \vec{n}+\vec{S}_{1} \cdot \vec{v}_{1} \dot{\vec{S}}_{1} \cdot \vec{n}+\dot{\vec{S}}_{1} \cdot \vec{v}_{1} \vec{S}_{1} \cdot \vec{n}\right. \\
& \left.\left.+\vec{S}_{1} \cdot \vec{a}_{1} \vec{S}_{1} \cdot \vec{n}\right)\right] \\
& -4 C_{1\left(E S^{2}\right)} \frac{G}{r^{2}} \frac{1}{m_{1}} \vec{S}_{2} \cdot \vec{v}_{2} \times \vec{n}\left(\dot{S}_{1}^{2}+\ddot{\vec{S}}_{1} \cdot \vec{S}_{1}\right),
\end{aligned}
$$

Fig. $2(\mathrm{a} 5)=-\frac{3}{2} C_{1\left(E S^{2}\right)} \frac{G}{r^{4}} \frac{1}{m_{1}}\left[2 \vec{S}_{1} \cdot \vec{S}_{2} \times \vec{v}_{1}\left(\vec{S}_{1} \cdot \vec{v}_{1} \vec{v}_{1} \cdot \vec{n}-\vec{S}_{1} \cdot \vec{n} v_{1}^{2}\right)\right.$

$$
\begin{aligned}
& -6 \vec{S}_{1} \cdot \vec{S}_{2} \times \vec{v}_{2} \vec{S}_{1} \cdot \vec{n} \vec{v}_{1} \cdot \vec{v}_{2}+\vec{S}_{2} \cdot \vec{v}_{1} \times \vec{v}_{2}\left(S_{1}^{2} \vec{v}_{2} \cdot \vec{n}+2 \vec{S}_{1} \cdot \vec{v}_{2} \vec{S}_{1} \cdot \vec{n}\right. \\
& \left.-5\left(\vec{S}_{1} \cdot \vec{n}\right)^{2} \vec{v}_{2} \cdot \vec{n}\right)-\vec{S}_{2} \cdot \vec{v}_{1} \times \vec{n}\left(S_{1}^{2}\left(3 v_{1}^{2}-10\left(\vec{v}_{1} \cdot \vec{n}\right)^{2}\right)-2\left(\vec{S}_{1} \cdot \vec{v}_{1}\right)^{2}\right. \\
& \left.+10 \vec{S}_{1} \cdot \vec{v}_{1} \vec{S}_{1} \cdot \vec{n} \vec{v}_{1} \cdot \vec{n}-5\left(\vec{S}_{1} \cdot \vec{n}\right)^{2} \vec{v}_{1}^{2}\right) \\
& \left.-3 \vec{S}_{2} \cdot \vec{v}_{2} \times \vec{n}\left(S_{1}^{2}-5\left(\vec{S}_{1} \cdot \vec{n}\right)^{2}\right) \vec{v}_{1} \cdot \vec{v}_{2}\right] \\
& -\frac{1}{2} C_{1\left(E S^{2}\right)} \frac{G}{r^{3}} \frac{1}{m_{1}}\left[\vec{S}_{1} \cdot \vec{S}_{2} \times \vec{v}_{2}\left(4 \dot{\vec{S}}_{1} \cdot \vec{v}_{2}-3 \dot{\vec{S}}_{1} \cdot \vec{n} \vec{v}_{2} \cdot \vec{n}\right)\right. \\
& +\overrightarrow{\vec{S}}_{1} \cdot \vec{S}_{2} \times \vec{v}_{2}\left(4 \vec{S}_{1} \cdot \vec{v}_{2}-3 \vec{S}_{1} \cdot \vec{n} \vec{v}_{2} \cdot \vec{n}\right)-3 \vec{S}_{1} \cdot \vec{S}_{2} \times \vec{n}\left(\vec{S}_{1} \cdot \vec{v}_{1} \vec{a}_{1} \cdot \vec{n}\right. \\
& \left.+\dot{\vec{S}}_{1} \cdot \vec{v}_{1} \vec{v}_{1} \cdot \vec{n}+\vec{S}_{1} \cdot \vec{a}_{1} \vec{v}_{1} \cdot \vec{n}-2 \vec{S}_{1} \cdot \vec{n} \vec{v}_{1} \cdot \vec{a}_{1}-\dot{\vec{S}}_{1} \cdot \vec{n} v_{1}^{2}\right) \\
& -3 \dot{\vec{S}}_{1} \cdot \vec{S}_{2} \times \vec{n}\left(\vec{S}_{1} \cdot \vec{v}_{1} \vec{v}_{1} \cdot \vec{n}-\vec{S}_{1} \cdot \vec{n} v_{1}^{2}\right) \\
& +4\left(\vec{S}_{2} \cdot \vec{v}_{1} \times \vec{a}_{2}+\dot{\vec{S}}_{2} \cdot \vec{v}_{1} \times \vec{v}_{2}\right)\left(S_{1}^{2}-3\left(\vec{S}_{1} \cdot \vec{n}\right)^{2}\right) \\
& -3 \vec{S}_{2} \cdot \vec{v}_{1} \times \vec{n}\left(2 S_{1}^{2} \vec{a}_{1} \cdot \vec{n}+4 \overrightarrow{\vec{S}}_{1} \cdot \vec{S}_{1} \vec{v}_{1} \cdot \vec{n}-\vec{S}_{1} \cdot \vec{v}_{1} \dot{\vec{S}}_{1} \cdot \vec{n}-\dot{\vec{S}}_{1} \cdot \vec{v}_{1} \vec{S}_{1} \cdot \vec{n}\right. \\
& \left.-\vec{S}_{1} \cdot \vec{a}_{1} \vec{S}_{1} \cdot \vec{n}\right)-3 \vec{S}_{2} \cdot \vec{a}_{1} \times \vec{n}\left(2 S_{1}^{2} \vec{v}_{1} \cdot \vec{n}-\vec{S}_{1} \cdot \vec{v}_{1} \vec{S}_{1} \cdot \vec{n}\right) \\
& \left.+3 \vec{S}_{2} \cdot \vec{v}_{2} \times \vec{n}\left(8 \dot{\vec{S}}_{1} \cdot \vec{S}_{1} \vec{v}_{2} \cdot \vec{n}-3 \vec{S}_{1} \cdot \vec{v}_{2} \dot{\vec{S}}_{1} \cdot \vec{n}-3 \dot{\vec{S}}_{1} \cdot \vec{v}_{2} \vec{S}_{1} \cdot \vec{n}\right)\right] \\
& +2 C_{1\left(E S^{2}\right)} \frac{G}{r^{2}} \frac{1}{m_{1}}\left[\left(\vec{S}_{1} \cdot \vec{S}_{2} \times \vec{a}_{2}+\vec{S}_{1} \cdot \dot{\vec{S}}_{2} \times \vec{v}_{2}\right) \dot{\vec{S}}_{1} \cdot \vec{n}\right. \\
& \left.+\left(\dot{\vec{S}}_{1} \cdot \dot{\vec{S}}_{2} \times \vec{v}_{2}+\dot{\vec{S}}_{1} \cdot \vec{S}_{2} \times \vec{a}_{2}\right) \vec{S}_{1} \cdot \vec{n}-2\left(\dot{\vec{S}}_{2} \cdot \vec{v}_{2} \times \vec{n}+\vec{S}_{2} \cdot \vec{a}_{2} \times \vec{n}\right) \dot{\vec{S}}_{1} \cdot \vec{S}_{1}\right]
\end{aligned}
$$

Fig. $2(\mathrm{a} 6)=-3 C_{1\left(E S^{2}\right)} \frac{G}{r^{4}} \frac{1}{m_{1}}\left[2 \vec{S}_{1} \cdot \vec{S}_{2} \times \vec{v}_{1} \vec{S}_{1} \cdot \vec{n} \vec{v}_{1} \cdot \vec{v}_{2}-2 \vec{S}_{1} \cdot \vec{S}_{2} \times \vec{v}_{2} \vec{S}_{1} \cdot \vec{n} v_{1}^{2}\right.$

$$
\begin{aligned}
& \left.+\left(\vec{S}_{2} \cdot \vec{v}_{1} \times \vec{n} \vec{v}_{1} \cdot \vec{v}_{2}-\vec{S}_{2} \cdot \vec{v}_{2} \times \vec{n} v_{1}^{2}\right)\left(S_{1}^{2}-5\left(\vec{S}_{1} \cdot \vec{n}\right)^{2}\right)\right] \\
& +C_{1\left(E S^{2}\right)} \frac{G}{m_{1} r^{3}}\left[\vec{S}_{1} \cdot \vec{S}_{2} \times \vec{v}_{1} \dot{\vec{S}}_{1} \cdot \vec{v}_{2}+\dot{\vec{S}}_{1} \cdot \vec{S}_{2} \times \vec{v}_{1} \vec{S}_{1} \cdot \vec{v}_{2}+2 \vec{S}_{1} \cdot \vec{S}_{2} \times \vec{a}_{1} \vec{S}_{1} \cdot \vec{v}_{2}\right. \\
& -2 \vec{S}_{1} \cdot \vec{S}_{2} \times \vec{v}_{2}\left(\dot{\vec{S}}_{1} \cdot \vec{v}_{1}+\vec{S}_{1} \cdot \vec{a}_{1}\right)-2 \dot{\vec{S}}_{1} \cdot \vec{S}_{2} \times \vec{v}_{2} \vec{S}_{1} \cdot \vec{v}_{1}
\end{aligned}
$$




$$
\begin{aligned}
& +3 \vec{S}_{1} \cdot \vec{S}_{2} \times \vec{n}\left(\vec{S}_{1} \cdot \vec{n} \vec{a}_{1} \cdot \vec{v}_{2}-\vec{S}_{1} \cdot \vec{v}_{2} \vec{a}_{1} \cdot \vec{n}+\dot{\vec{S}}_{1} \cdot \vec{n} \vec{v}_{1} \cdot \vec{v}_{2}\right) \\
& +3 \dot{\vec{S}}_{1} \cdot \vec{S}_{2} \times \vec{n} \vec{S}_{1} \cdot \vec{n} \vec{v}_{1} \cdot \vec{v}_{2}-6 \vec{S}_{2} \cdot \vec{v}_{1} \times \vec{v}_{2} \cdot \overrightarrow{\vec{S}}_{1}+2 \vec{S}_{2} \cdot \vec{v}_{2} \times \vec{a}_{1} S_{1}^{2} \\
& +3 \vec{S}_{2} \cdot \vec{v}_{1} \times \vec{n}\left(2 \dot{\vec{S}}_{1} \cdot \vec{S}_{1} \vec{v}_{2} \cdot \vec{n}-\vec{S}_{1} \cdot \vec{v}_{2} \dot{\vec{S}}_{1} \cdot \vec{n}-\dot{\vec{S}}_{1} \cdot \vec{v}_{2} \vec{S}_{1} \cdot \vec{n}\right) \\
& -3 \vec{S}_{2} \cdot \vec{v}_{2} \times \vec{n}\left(S_{1}^{2} \vec{a}_{1} \cdot \vec{n}+4 \dot{\vec{S}}_{1} \cdot \vec{S}_{1} \vec{v}_{1} \cdot \vec{n}\right. \\
& \left.-2 \vec{S}_{1} \cdot \vec{v}_{1} \dot{\vec{S}}_{1} \cdot \vec{n}-2 \dot{\vec{S}}_{1} \cdot \vec{v}_{1} \vec{S}_{1} \cdot \vec{n}-2 \vec{S}_{1} \cdot \vec{n} \vec{S}_{1} \cdot \vec{a}_{1}\right) \\
& \left.+3 \vec{S}_{2} \cdot \vec{a}_{1} \times \vec{n}\left(S_{1}^{2} \vec{v}_{2} \cdot \vec{n}-\vec{S}_{1} \cdot \vec{v}_{2} \vec{S}_{1} \cdot \vec{n}\right)\right] \\
& -C_{1\left(E S^{2}\right)} \frac{G}{r^{2}} \frac{1}{m_{1}}\left[\vec{S}_{1} \cdot \vec{S}_{2} \times \vec{n} \ddot{\vec{S}}_{1} \cdot \vec{v}_{2}+2 \dot{\vec{S}}_{1} \cdot \vec{S}_{2} \times \vec{n} \dot{\vec{S}}_{1} \cdot \vec{v}_{2}\right. \\
& \left.+\ddot{\vec{S}}_{1} \cdot \vec{S}_{2} \times \vec{n} \vec{S}_{1} \cdot \vec{v}_{2}-2 \vec{S}_{2} \cdot \vec{v}_{2} \times \vec{n}\left(\dot{S}_{1}^{2}+\ddot{\vec{S}}_{1} \cdot \vec{S}_{1}\right)\right],
\end{aligned}
$$

Fig. $2(\mathrm{a} 7)=\frac{1}{2} C_{1\left(B S^{3}\right)} \frac{G}{r^{4}} \frac{m_{2}}{m_{1}^{2}}\left[\vec{S}_{1} \cdot \vec{v}_{1} \times \vec{v}_{2}\left(S_{1}^{2} \vec{v}_{2} \cdot \vec{n}+2 \vec{S}_{1} \cdot \vec{v}_{2} \vec{S}_{1} \cdot \vec{n}-5\left(\vec{S}_{1} \cdot \vec{n}\right)^{2} \vec{v}_{2} \cdot \vec{n}\right)\right.$

$-\vec{S}_{1} \cdot \vec{v}_{2} \times \vec{n}\left(S_{1}^{2}\left(\vec{v}_{1} \cdot \vec{v}_{2}-5 \vec{v}_{1} \cdot \vec{n} \vec{v}_{2} \cdot \vec{n}\right)+2 \vec{S}_{1} \cdot \vec{v}_{1} \vec{S}_{1} \cdot \vec{v}_{2}\right.$

$-10 \vec{S}_{1} \cdot \vec{v}_{1} \vec{S}_{1} \cdot \vec{n} \vec{v}_{2} \cdot \vec{n}-10 \vec{S}_{1} \cdot \vec{v}_{2} \vec{S}_{1} \cdot \vec{n} \vec{v}_{1} \cdot \vec{n}$

$$
\begin{aligned}
& \left.\left.-5\left(\vec{S}_{1} \cdot \vec{n}\right)^{2}\left(\vec{v}_{1} \cdot \vec{v}_{2}-7 \vec{v}_{1} \cdot \vec{n} \vec{v}_{2} \cdot \vec{n}\right)\right)\right] \\
& +\frac{1}{6} C_{1\left(B S^{3}\right)} \frac{G}{r^{3}} \frac{m_{2}}{m_{1}^{2}}\left[\vec{S}_{1} \cdot \vec{v}_{1} \times \vec{a}_{2}\left(S_{1}^{2}-3\left(\vec{S}_{1} \cdot \vec{n}\right)^{2}\right)-6 \vec{S}_{1} \cdot \vec{v}_{2} \times \vec{n}\left(\dot{\vec{S}}_{1} \cdot \vec{S}_{1} \vec{v}_{2} \cdot \vec{n}\right.\right.
\end{aligned}
$$$$
\left.+\dot{\vec{S}}_{1} \cdot \vec{v}_{2} \vec{S}_{1} \cdot \vec{n}+\vec{S}_{1} \cdot \vec{v}_{2} \dot{\vec{S}}_{1} \cdot \vec{n}-5 \dot{\vec{S}}_{1} \cdot \vec{n} \vec{S}_{1} \cdot \vec{n} \vec{v}_{2} \cdot \vec{n}\right)
$$$$
+3 \vec{S}_{1} \cdot \vec{a}_{2} \times \vec{n}\left(S_{1}^{2} \vec{v}_{1} \cdot \vec{n}+2 \vec{S}_{1} \cdot \vec{v}_{1} \vec{S}_{1} \cdot \vec{n}-5\left(\vec{S}_{1} \cdot \vec{n}\right)^{2} \vec{v}_{1} \cdot \vec{n}\right)
$$$$
\left.-3 \dot{\vec{S}}_{1} \cdot \vec{v}_{2} \times \vec{n}\left(S_{1}^{2} \vec{v}_{2} \cdot \vec{n}+2 \vec{S}_{1} \cdot \vec{v}_{2} \vec{S}_{1} \cdot \vec{n}-5\left(\vec{S}_{1} \cdot \vec{n}\right)^{2} \vec{v}_{2} \cdot \vec{n}\right)\right]
$$$$
-\frac{1}{6} C_{1\left(B S^{3}\right)} \frac{G}{r^{2}} \frac{m_{2}}{m_{1}^{2}}\left[2 \vec{S}_{1} \cdot \vec{a}_{2} \times \vec{n}\left(\dot{\vec{S}}_{1} \cdot \vec{S}_{1}-3 \dot{\vec{S}}_{1} \cdot \vec{n} \vec{S}_{1} \cdot \vec{n}\right)\right.
$$$$
\left.+\dot{\vec{S}}_{1} \cdot \vec{a}_{2} \times \vec{n}\left(S_{1}^{2}-3\left(\vec{S}_{1} \cdot \vec{n}\right)^{2}\right)\right],
$$

Fig. $2(\mathrm{a} 8)=\frac{1}{2} C_{1\left(B S^{3}\right)} \frac{G}{r^{4}} \frac{m_{2}}{m_{1}^{2}}\left[\vec{S}_{1} \cdot \vec{v}_{1} \times \vec{v}_{2}\left(S_{1}^{2} \vec{v}_{1} \cdot \vec{n}+2 \vec{S}_{1} \cdot \vec{v}_{1} \vec{S}_{1} \cdot \vec{n}-5\left(\vec{S}_{1} \cdot \vec{n}\right)^{2} \vec{v}_{1} \cdot \vec{n}\right)\right.$

$+\vec{S}_{1} \cdot \vec{v}_{1} \times \vec{n}\left(S_{1}^{2}\left(\vec{v}_{1} \cdot \vec{v}_{2}-5 \vec{v}_{1} \cdot \vec{n} \vec{v}_{2} \cdot \vec{n}\right)+2 \vec{S}_{1} \cdot \vec{v}_{1} \vec{S}_{1} \cdot \vec{v}_{2}\right.$

$-10 \vec{S}_{1} \cdot \vec{v}_{1} \vec{S}_{1} \cdot \vec{n} \vec{v}_{2} \cdot \vec{n}-10 \vec{S}_{1} \cdot \vec{v}_{2} \vec{S}_{1} \cdot \vec{n} \vec{v}_{1} \cdot \vec{n}$

$\left.\left.-5\left(\vec{S}_{1} \cdot \vec{n}\right)^{2}\left(\vec{v}_{1} \cdot \vec{v}_{2}-7 \vec{v}_{1} \cdot \vec{n} \vec{v}_{2} \cdot \vec{n}\right)\right)\right]$

$-\frac{1}{6} C_{1\left(B S^{3}\right)} \frac{G}{r^{3}} \frac{m_{2}}{m_{1}^{2}}\left[2 \vec{S}_{1} \cdot \vec{v}_{1} \times \vec{v}_{2}\left(\dot{\vec{S}}_{1} \cdot \vec{S}_{1}-3 \dot{\vec{S}}_{1} \cdot \vec{n} \vec{S}_{1} \cdot \vec{n}\right)\right.$

$-\left(\vec{S}_{1} \cdot \vec{v}_{2} \times \vec{a}_{1}-\dot{\vec{S}}_{1} \cdot \vec{v}_{1} \times \vec{v}_{2}\right)\left(S_{1}^{2}-3\left(\vec{S}_{1} \cdot \vec{n}\right)^{2}\right)-6 \vec{S}_{1} \cdot \vec{v}_{1} \times \vec{n}\left(\dot{\vec{S}}_{1} \cdot \vec{S}_{1} \vec{v}_{2} \cdot \vec{n}\right.$

$\left.+\dot{\vec{S}}_{1} \cdot \vec{v}_{2} \vec{S}_{1} \cdot \vec{n}+\vec{S}_{1} \cdot \vec{v}_{2} \dot{\vec{S}}_{1} \cdot \vec{n}-5 \dot{\vec{S}}_{1} \cdot \vec{n} \vec{S}_{1} \cdot \vec{n} \vec{v}_{2} \cdot \vec{n}\right)$

$\left.-3\left(\vec{S}_{1} \cdot \vec{a}_{1} \times \vec{n}+\dot{\vec{S}}_{1} \cdot \vec{v}_{1} \times \vec{n}\right)\left(S_{1}^{2} \vec{v}_{2} \cdot \vec{n}+2 \vec{S}_{1} \cdot \vec{v}_{2} \vec{S}_{1} \cdot \vec{n}-5\left(\vec{S}_{1} \cdot \vec{n}\right)^{2} \vec{v}_{2} \cdot \vec{n}\right)\right]$, 
Fig. $2(\mathrm{a} 9)=-\frac{3}{2} C_{1\left(E S^{2}\right)} \frac{G}{r^{4}} \frac{1}{m_{1}}\left[2 \vec{S}_{1} \cdot \vec{S}_{2} \times \vec{v}_{2}\left(\vec{S}_{1} \cdot \vec{v}_{1} \vec{v}_{2} \cdot \vec{n}+\vec{S}_{1} \cdot \vec{v}_{2} \vec{v}_{1} \cdot \vec{n}+\vec{S}_{1} \cdot \vec{n} \vec{v}_{1} \cdot \vec{v}_{2}\right.\right.$ $\left.-5 \vec{S}_{1} \cdot \vec{n} \vec{v}_{1} \cdot \vec{n} \vec{v}_{2} \cdot \vec{n}\right)-\vec{S}_{2} \cdot \vec{v}_{1} \times \vec{v}_{2}\left(S_{1}^{2} \vec{v}_{2} \cdot \vec{n}+2 \vec{S}_{1} \cdot \vec{v}_{2} \vec{S}_{1} \cdot \vec{n}\right.$ $\left.-5\left(\vec{S}_{1} \cdot \vec{n}\right)^{2} \vec{v}_{2} \cdot \vec{n}\right)-\vec{S}_{2} \cdot \vec{v}_{2} \times \vec{n}\left(S_{1}^{2}\left(\vec{v}_{1} \cdot \vec{v}_{2}-5 \vec{v}_{1} \cdot \vec{n} \vec{v}_{2} \cdot \vec{n}\right)\right.$ $\left.-2 \vec{S}_{1} \cdot \vec{v}_{1} \vec{S}_{1} \cdot \vec{v}_{2}+10 \vec{S}_{1} \cdot \vec{v}_{1} \vec{S}_{1} \cdot \vec{n} \vec{v}_{2} \cdot \vec{n}+10 \vec{S}_{1} \cdot \vec{v}_{2} \vec{S}_{1} \cdot \vec{n} \vec{v}_{1} \cdot \vec{n}\right)$ $\left.\left.+5\left(\vec{S}_{1} \cdot \vec{n}\right)^{2}\left(\vec{v}_{1} \cdot \vec{v}_{2}-7 \vec{v}_{1} \cdot \vec{n} \vec{v}_{2} \cdot \vec{n}\right)\right)\right]$ $+\frac{1}{2} C_{1\left(E S^{2}\right)} \frac{G}{r^{3}} \frac{1}{m_{1}}\left[2 \vec{S}_{1} \cdot \vec{S}_{2} \times \vec{v}_{2}\left(\dot{\vec{S}}_{1} \cdot \vec{v}_{2}-3 \dot{\vec{S}}_{1} \cdot \vec{n} \vec{v}_{2} \cdot \vec{n}\right)\right.$ $-2\left(\vec{S}_{1} \cdot \vec{S}_{2} \times \vec{a}_{2}+\vec{S}_{1} \cdot \dot{\vec{S}}_{2} \times \vec{v}_{2}\right)\left(\vec{S}_{1} \cdot \vec{v}_{1}-3 \vec{S}_{1} \cdot \vec{n} \vec{v}_{1} \cdot \vec{n}\right)$ $+2 \dot{\vec{S}}_{1} \cdot \vec{S}_{2} \times \vec{v}_{2}\left(\vec{S}_{1} \cdot \vec{v}_{2}-3 \vec{S}_{1} \cdot \vec{n} \vec{v}_{2} \cdot \vec{n}\right)$ $-\left(\vec{S}_{2} \cdot \vec{v}_{1} \times \vec{a}_{2}+\dot{\vec{S}}_{2} \cdot \vec{v}_{1} \times \vec{v}_{2}\right)\left(S_{1}^{2}+3\left(\vec{S}_{1} \cdot \vec{n}\right)^{2}\right)+6 \vec{S}_{2} \cdot \vec{v}_{2} \times \vec{n}\left(\dot{\vec{S}}_{1} \cdot \vec{S}_{1} \vec{v}_{2} \cdot \vec{n}\right.$ $\left.-\dot{\vec{S}}_{1} \cdot \vec{v}_{2} \vec{S}_{1} \cdot \vec{n}-\vec{S}_{1} \cdot \vec{v}_{2} \dot{\vec{S}}_{1} \cdot \vec{n}+5 \dot{\vec{S}}_{1} \cdot \vec{n} \vec{S}_{1} \cdot \vec{n} \vec{v}_{2} \cdot \vec{n}\right)$ $\left.-3\left(\dot{\vec{S}}_{2} \cdot \vec{v}_{2} \times \vec{n}+\vec{S}_{2} \cdot \vec{a}_{2} \times \vec{n}\right)\left(S_{1}^{2} \vec{v}_{1} \cdot \vec{n}-2 \vec{S}_{1} \cdot \vec{v}_{1} \vec{S}_{1} \cdot \vec{n}+5\left(\vec{S}_{1} \cdot \vec{n}\right)^{2} \vec{v}_{1} \cdot \vec{n}\right)\right]$ $-C_{1\left(E S^{2}\right)} \frac{G}{r^{2}} \frac{1}{m_{1}}\left[\vec{S}_{1} \cdot \vec{S}_{2} \times \vec{a}_{2} \dot{\vec{S}}_{1} \cdot \vec{n}+\vec{S}_{1} \cdot \dot{\vec{S}}_{2} \times \vec{v}_{2} \dot{\vec{S}}_{1} \cdot \vec{n}+\dot{\vec{S}}_{1} \cdot \vec{S}_{2} \times \vec{a}_{2} \vec{S}_{1} \cdot \vec{n}\right.$ $\left.+\dot{\vec{S}}_{1} \cdot \dot{\vec{S}}_{2} \times \vec{v}_{2} \vec{S}_{1} \cdot \vec{n}-\left(\dot{\vec{S}}_{2} \cdot \vec{v}_{2} \times \vec{n}+\vec{S}_{2} \cdot \vec{a}_{2} \times \vec{n}\right)\left(\dot{\vec{S}}_{1} \cdot \vec{S}_{1}+3 \dot{\vec{S}}_{1} \cdot \vec{n} \vec{S}_{1} \cdot \vec{n}\right)\right]$,

Fig. $2(\mathrm{a} 10)=\frac{3}{2} C_{1\left(E S^{2}\right)} \frac{G}{r^{4}} \frac{1}{m_{1}}\left[2 \vec{S}_{1} \cdot \vec{S}_{2} \times \vec{v}_{1}\left(\vec{S}_{1} \cdot \vec{v}_{1} \vec{v}_{2} \cdot \vec{n}+\vec{S}_{1} \cdot \vec{v}_{2} \vec{v}_{1} \cdot \vec{n}+\overrightarrow{S_{1}} \cdot \vec{n} \vec{v}_{1} \cdot \vec{v}_{2}\right.\right.$

$\left.-5 \vec{S}_{1} \cdot \vec{n} \vec{v}_{1} \cdot \vec{n} \vec{v}_{2} \cdot \vec{n}\right)-\vec{S}_{2} \cdot \vec{v}_{1} \times \vec{v}_{2}\left(S_{1}^{2} \vec{v}_{1} \cdot \vec{n}-2 \vec{S}_{1} \cdot \vec{v}_{1} \vec{S}_{1} \cdot \vec{n}\right.$

$\left.+5\left(\vec{S}_{1} \cdot \vec{n}\right)^{2} \vec{v}_{1} \cdot \vec{n}\right)-\vec{S}_{2} \cdot \vec{v}_{1} \times \vec{n}\left(S_{1}^{2}\left(\vec{v}_{1} \cdot \vec{v}_{2}-5 \vec{v}_{1} \cdot \vec{n} \vec{v}_{2} \cdot \vec{n}\right)\right.$

$-2 \vec{S}_{1} \cdot \vec{v}_{1} \vec{S}_{1} \cdot \vec{v}_{2}+10 \vec{S}_{1} \cdot \vec{v}_{1} \vec{S}_{1} \cdot \vec{n} \vec{v}_{2} \cdot \vec{n}+10 \vec{S}_{1} \cdot \vec{v}_{2} \vec{S}_{1} \cdot \vec{n} \vec{v}_{1} \cdot \vec{n}$

$\left.\left.+5\left(\vec{S}_{1} \cdot \vec{n}\right)^{2}\left(\vec{v}_{1} \cdot \vec{v}_{2}-7 \vec{v}_{1} \cdot \vec{n} \vec{v}_{2} \cdot \vec{n}\right)\right)\right]$

$-\frac{1}{2} C_{1\left(E S^{2}\right)} \frac{G}{r^{3}} \frac{1}{m_{1}}\left[\vec{S}_{1} \cdot \vec{S}_{2} \times \vec{v}_{1}\left(\dot{\vec{S}}_{1} \cdot \vec{v}_{2}-3 \dot{\vec{S}}_{1} \cdot \vec{n} \vec{v}_{2} \cdot \vec{n}\right)\right.$

$+2 \vec{S}_{1} \cdot \vec{S}_{2} \times \vec{a}_{1}\left(\vec{S}_{1} \cdot \vec{v}_{2}-3 \vec{S}_{1} \cdot \vec{n} \vec{v}_{2} \cdot \vec{n}\right)+\dot{\vec{S}}_{1} \cdot \vec{S}_{2} \times \vec{v}_{1}\left(\vec{S}_{1} \cdot \vec{v}_{2}-3 \vec{S}_{1} \cdot \vec{n} \vec{v}_{2} \cdot \vec{n}\right)$

$-2 \vec{S}_{1} \cdot \dot{\vec{S}}_{2} \times \vec{v}_{1}\left(\vec{S}_{1} \cdot \vec{v}_{1}-3 \vec{S}_{1} \cdot \vec{n} \vec{v}_{1} \cdot \vec{n}\right)-\vec{S}_{1} \cdot \vec{S}_{2} \times \vec{v}_{2}\left(\dot{\vec{S}}_{1} \cdot \vec{v}_{1}-3 \dot{\vec{S}}_{1} \cdot \vec{n} \vec{v}_{1} \cdot \vec{n}\right)$

$-\dot{\vec{S}}_{1} \cdot \vec{S}_{2} \times \vec{v}_{2}\left(\vec{S}_{1} \cdot \vec{v}_{1}-3 \vec{S}_{1} \cdot \vec{n} \vec{v}_{1} \cdot \vec{n}\right)+3 \vec{S}_{1} \cdot \vec{S}_{2} \times \vec{n}\left(\dot{\vec{S}}_{1} \cdot \vec{v}_{1} \vec{v}_{2} \cdot \vec{n}\right.$

$\left.+\dot{\vec{S}}_{1} \cdot \vec{v}_{2} \vec{v}_{1} \cdot \vec{n}+\dot{\vec{S}}_{1} \cdot \vec{n} \vec{v}_{1} \cdot \vec{v}_{2}-5 \dot{\vec{S}}_{1} \cdot \vec{n} \vec{v}_{1} \cdot \vec{n} \vec{v}_{2} \cdot \vec{n}\right)$

$+3 \dot{\vec{S}}_{1} \cdot \vec{S}_{2} \times \vec{n}\left(\vec{S}_{1} \cdot \vec{v}_{1} \vec{v}_{2} \cdot \vec{n}+\vec{S}_{1} \cdot \vec{v}_{2} \vec{v}_{1} \cdot \vec{n}+\vec{S}_{1} \cdot \vec{n} \vec{v}_{1} \cdot \vec{v}_{2}\right.$

$\left.-5 \vec{S}_{1} \cdot \vec{n} \vec{v}_{1} \cdot \vec{n} \vec{v}_{2} \cdot \vec{n}\right)-2 \vec{S}_{2} \cdot \vec{v}_{1} \times \vec{v}_{2}\left(\dot{\vec{S}}_{1} \cdot \vec{S}_{1}+3 \dot{\vec{S}}_{1} \cdot \vec{n} \vec{S}_{1} \cdot \vec{n}\right)$

$-\vec{S}_{2} \cdot \vec{a}_{1} \times \vec{v}_{2}\left(S_{1}^{2}+3\left(\vec{S}_{1} \cdot \vec{n}\right)^{2}\right)+6 \vec{S}_{2} \cdot \vec{v}_{1} \times \vec{n}\left(\dot{\vec{S}}_{1} \cdot \vec{S}_{1} \vec{v}_{2} \cdot \vec{n}-\vec{S}_{1} \cdot \vec{v}_{2} \dot{\vec{S}}_{1} \cdot \vec{n}\right.$ 


$$
\begin{aligned}
& \left.-\dot{\vec{S}}_{1} \cdot \vec{v}_{2} \vec{S}_{1} \cdot \vec{n}+5 \dot{\vec{S}}_{1} \cdot \vec{n} \vec{S}_{1} \cdot \vec{n} \vec{v}_{2} \cdot \vec{n}\right) \\
& +3 \vec{S}_{2} \cdot \vec{a}_{1} \times \vec{n}\left(S_{1}^{2} \vec{v}_{2} \cdot \vec{n}-2 \vec{S}_{1} \cdot \vec{v}_{2} \vec{S}_{1} \cdot \vec{n}+5\left(\vec{S}_{1} \cdot \vec{n}\right)^{2} \vec{v}_{2} \cdot \vec{n}\right) \\
& \left.-3 \dot{\vec{S}}_{2} \cdot \vec{v}_{1} \times \vec{n}\left(S_{1}^{2} \vec{v}_{1} \cdot \vec{n}-2 \vec{S}_{1} \cdot \vec{v}_{1} \vec{S}_{1} \cdot \vec{n}+5\left(\vec{S}_{1} \cdot \vec{n}\right)^{2} \vec{v}_{1} \cdot \vec{n}\right)\right] \\
& +\frac{1}{2} C_{1\left(E S^{2}\right)} \frac{G}{r^{2}} \frac{1}{m_{1}}\left[\vec{S}_{1} \cdot \dot{\vec{S}}_{2} \times \vec{v}_{1} \dot{\vec{S}}_{1} \cdot \vec{n}+\dot{\vec{S}}_{1} \cdot \dot{\vec{S}}_{2} \times \vec{v}_{1} \vec{S}_{1} \cdot \vec{n}\right. \\
& +\vec{S}_{1} \cdot \vec{S}_{2} \times \vec{v}_{2} \ddot{\vec{S}}_{1} \cdot \vec{n}+2 \dot{\vec{S}}_{1} \cdot \vec{S}_{2} \times \vec{v}_{2} \dot{\vec{S}}_{1} \cdot \vec{n}+\ddot{\vec{S}}_{1} \cdot \vec{S}_{2} \times \vec{v}_{2} \vec{S}_{1} \cdot \vec{n} \\
& +\vec{S}_{1} \cdot \vec{S}_{2} \times \vec{n}\left(\ddot{\vec{S}}_{1} \cdot \vec{v}_{2}-3 \ddot{\vec{S}}_{1} \cdot \vec{n} \vec{v}_{2} \cdot \vec{n}\right)-\vec{S}_{1} \cdot \dot{\vec{S}}_{2} \times \vec{n}\left(\dot{\vec{S}}_{1} \cdot \vec{v}_{1}-3 \dot{\vec{S}}_{1} \cdot \vec{n} \vec{v}_{1} \cdot \vec{n}\right) \\
& +2 \dot{\vec{S}}_{1} \cdot \vec{S}_{2} \times \vec{n}\left(\dot{\vec{S}}_{1} \cdot \vec{v}_{2}-3 \dot{\vec{S}}_{1} \cdot \vec{n} \vec{v}_{2} \cdot \vec{n}\right)-\dot{\vec{S}}_{1} \cdot \dot{\vec{S}}_{2} \times \vec{n}\left(\vec{S}_{1} \cdot \vec{v}_{1}-3 \vec{S}_{1} \cdot \vec{n} \vec{v}_{1} \cdot \vec{n}\right) \\
& +\ddot{\vec{S}}_{1} \cdot \vec{S}_{2} \times \vec{n}\left(\vec{S}_{1} \cdot \vec{v}_{2}-3 \vec{S}_{1} \cdot \vec{n} \vec{v}_{2} \cdot \vec{n}\right)+2 \vec{S}_{1} \cdot \dot{\vec{S}}_{2} \times \vec{a}_{1} \vec{S}_{1} \cdot \vec{n} \\
& \left.-2 \dot{\vec{S}}_{2} \cdot \vec{v}_{1} \times \vec{n}\left(\dot{\vec{S}}_{1} \cdot \vec{S}_{1}+3 \dot{\vec{S}}_{1} \cdot \vec{n} \vec{S}_{1} \cdot \vec{n}\right)-\dot{\vec{S}}_{2} \cdot \vec{a}_{1} \times \vec{n}\left(S_{1}^{2}+3\left(\vec{S}_{1} \cdot \vec{n}\right)^{2}\right)\right] \\
& -\frac{1}{2} C_{1\left(E S^{2}\right)} \frac{G}{r} \frac{1}{m_{1}}\left[\vec{S}_{1} \cdot \dot{\vec{S}}_{2} \times \vec{n} \ddot{\vec{S}}_{1} \cdot \vec{n}+2 \dot{\vec{S}}_{1} \cdot \dot{\vec{S}}_{2} \times \vec{n} \dot{\vec{S}}_{1} \cdot \vec{n}+\ddot{\vec{S}}_{1} \cdot \dot{\vec{S}}_{2} \times \vec{n} \vec{S}_{1} \cdot \vec{n}\right] .
\end{aligned}
$$

Note that almost all these graphs contain higher order time derivatives terms, notably second order time derivatives, where graph 1 (a10) even contains third order ones. Notice also that the value of graph 1(a5) will have to be supplemented with a piece that contains time derivatives of the spin, that appeared already in graph 2(a) of the LO in [11], but eventually did not contribute at the LO. At this order, as we will see here in section 5 these terms actually contribute.

\subsection{Two-graviton exchange}

As can be seen in figure 3 we have 15 graphs of two-graviton exchange in this sector. Here the majority of the graphs do not involve time derivatives. We have here two new two-graviton octupole couplings in graphs 1(b1), 1(b2), and on the other hand we have here nonlinearities originating from gravitons sourced strictly from minimal coupling to the worldline as in graphs 1(b13)-1(b15).

The graphs in figure 3 are evaluated as follows:

Fig. $3(\mathrm{~b} 1)=C_{1\left(B S^{3}\right)} \frac{G^{2}}{r^{5}} \frac{m_{2}^{2}}{m_{1}^{2}} \vec{S}_{1} \cdot \vec{v}_{2} \times \vec{n}\left[9 S_{1}^{2}-50\left(\vec{S}_{1} \cdot \vec{n}\right)^{2}\right]$,

Fig. $3(\mathrm{~b} 2)=-\frac{1}{3} C_{1\left(B S^{3}\right)} \frac{G^{2}}{r^{5}} \frac{m_{2}^{2}}{m_{1}^{2}} \vec{S}_{1} \cdot \vec{v}_{1} \times \vec{n}\left[11 S_{1}^{2}-54\left(\vec{S}_{1} \cdot \vec{n}\right)^{2}\right]$,

Fig. $3(\mathrm{~b} 3)=C_{1\left(B S^{3}\right)} \frac{G^{2}}{r^{5}} \frac{m_{2}}{m_{1}} \vec{S}_{1} \cdot \vec{v}_{2} \times \vec{n}\left[S_{1}^{2}-5\left(\vec{S}_{1} \cdot \vec{n}\right)^{2}\right]$,

Fig. $3(\mathrm{~b} 4)=-C_{1\left(B S^{3}\right)} \frac{G^{2}}{r^{5}} \frac{m_{2}}{m_{1}} \vec{S}_{1} \cdot \vec{v}_{1} \times \vec{n}\left[S_{1}^{2}-5\left(\vec{S}_{1} \cdot \vec{n}\right)^{2}\right]$,

Fig. $3($ b5 $)=8 C_{1\left(E S^{2}\right)} \frac{G^{2}}{r^{5}} \frac{m_{2}}{m_{1}}\left[3 \vec{S}_{1} \cdot \vec{S}_{2} \times \vec{v}_{2} \vec{S}_{1} \cdot \vec{n}+\vec{S}_{2} \cdot \vec{v}_{2} \times \vec{n}\left[2 S_{1}^{2}-9\left(\vec{S}_{1} \cdot \vec{n}\right)^{2}\right]\right]$, 


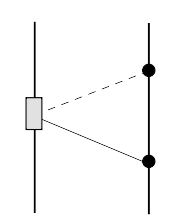

(b1)

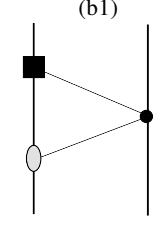

(b9)

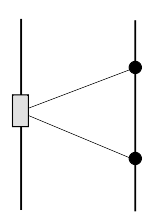

(b2)

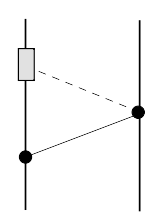

(b3)

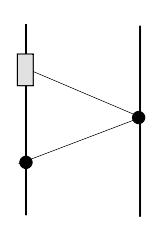

(b4)

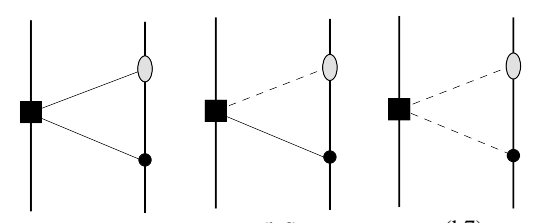

(b7)

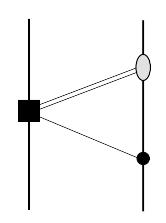

(b8)

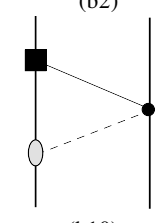

(b10)

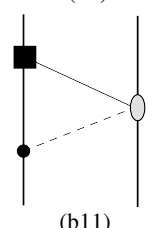

(b11)

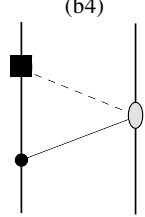

(b12)

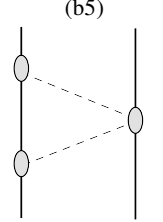

(b13)

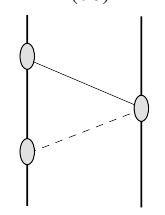

(b14)

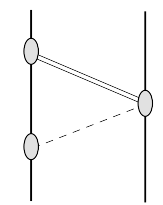

(b15)

Figure 3. The Feynman graphs of two-graviton exchange, which contribute to the NLO cubic-inspin interaction at the $4.5 \mathrm{PN}$ order for maximally-rotating compact objects. The graphs should be included together with their mirror images, i.e. with the worldline labels $1 \leftrightarrow 2$ exchanged. These graphs include all relevant interactions among the spin-induced quadrupole, octupole, and the mass and spin, in particular here at the nonlinear level there are also interactions involving the various multipoles on two different points of the worldline, which add up to interactions that are cubic in the spin, such as a spin dipole and a spin-induced quadrupole or two spin dipoles, on the same worldline, which can already be seen as of the NLO spin-squared sector $[12,14]$. Consequently notice that there are nonlinearities originating from gravitons sourced strictly from minimal coupling to the worldline as shown in graphs (b13)-(b15). We also have here two new two-graviton octupole couplings in graphs (b1), (b2).

Fig. $3(\mathrm{~b} 6)=C_{1\left(E S^{2}\right)} \frac{G^{2}}{r^{5}} \frac{m_{2}}{m_{1}}\left[-23 \vec{S}_{1} \cdot \vec{S}_{2} \times \vec{v}_{1} \vec{S}_{1} \cdot \vec{n}+13 \vec{S}_{1} \cdot \vec{S}_{2} \times \vec{v}_{2} \vec{S}_{1} \cdot \vec{n}\right.$

$$
\begin{aligned}
& -\vec{S}_{2} \cdot \vec{v}_{1} \times \vec{n}\left(31 S_{1}^{2}-66\left(\vec{S}_{1} \cdot \vec{n}\right)^{2}\right)-\vec{S}_{1} \cdot \vec{S}_{2} \times \vec{n}\left(10 \vec{S}_{1} \cdot \vec{v}_{1}-51 \vec{S}_{1} \cdot \vec{n} \vec{v}_{1} \cdot \vec{n}\right) \\
& \left.+\vec{S}_{1} \cdot \vec{S}_{2} \times \vec{n}\left(11 \vec{S}_{1} \cdot \vec{v}_{2}-54 \vec{S}_{1} \cdot \vec{n} \vec{v}_{2} \cdot \vec{n}\right)\right] \\
& -13 C_{1\left(E S^{2}\right)} \frac{G^{2}}{r^{4}} \frac{m_{2}}{m_{1}}\left[\vec{S}_{1} \cdot \dot{\vec{S}}_{2} \times \vec{n} \vec{S}_{1} \cdot \vec{n}\right],
\end{aligned}
$$

Fig. $3(\mathrm{~b} 7)=2 C_{1\left(E S^{2}\right)} \frac{G^{2}}{r^{5}} \frac{m_{2}}{m_{1}}\left[2 \vec{S}_{1} \cdot \vec{S}_{2} \times \vec{v}_{2} \vec{S}_{1} \cdot \vec{n}\right.$

$$
\left.+\vec{S}_{1} \cdot \vec{S}_{2} \times \vec{n}\left(\vec{S}_{1} \cdot \vec{v}_{2}-3 \vec{S}_{1} \cdot \vec{n} \vec{v}_{2} \cdot \vec{n}\right)+\vec{S}_{2} \cdot \vec{v}_{2} \times \vec{n}\left(2 S_{1}^{2}-3\left(\vec{S}_{1} \cdot \vec{n}\right)^{2}\right)\right],
$$

Fig. $3(\mathrm{~b} 8)=-C_{1\left(E S^{2}\right)} \frac{G^{2}}{r^{5}} \frac{m_{2}}{m_{1}}\left[2 \vec{S}_{1} \cdot \vec{S}_{2} \times \vec{v}_{2} \vec{S}_{1} \cdot \vec{n}\right.$

$$
\left.+3 \vec{S}_{1} \cdot \vec{S}_{2} \times \vec{n}\left(\vec{S}_{1} \cdot \vec{v}_{2}-2 \vec{S}_{1} \cdot \vec{n} \vec{v}_{2} \cdot \vec{n}\right)+\vec{S}_{2} \cdot \vec{v}_{2} \times \vec{n}\left(5 S_{1}^{2}-12\left(\vec{S}_{1} \cdot \vec{n}\right)^{2}\right)\right],
$$

Fig. 3(b9) $=-C_{1\left(E S^{2}\right)} \frac{G^{2}}{r^{5}} \frac{m_{2}}{m_{1}} \vec{S}_{1} \cdot \vec{v}_{1} \times \vec{n}\left[S_{1}^{2}-3\left(\vec{S}_{1} \cdot \vec{n}\right)^{2}\right]$,

Fig. 3(b10) $=C_{1\left(E S^{2}\right)} \frac{G^{2}}{r^{5}} \frac{m_{2}}{m_{1}} \vec{S}_{1} \cdot \vec{v}_{2} \times \vec{n}\left[S_{1}^{2}-3\left(\vec{S}_{1} \cdot \vec{n}\right)^{2}\right]$,

Fig. $3(\mathrm{~b} 11)=-4 C_{1\left(E S^{2}\right)} \frac{G^{2}}{r^{5}} \vec{S}_{2} \cdot \vec{v}_{1} \times \vec{n}\left[S_{1}^{2}-3\left(\vec{S}_{1} \cdot \vec{n}\right)^{2}\right]$, 


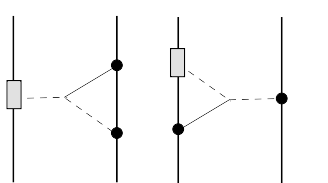

(a1)

(a2)

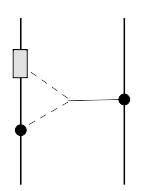

(a3)

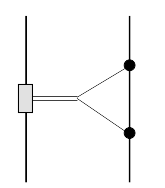

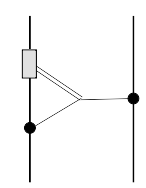

(a5)

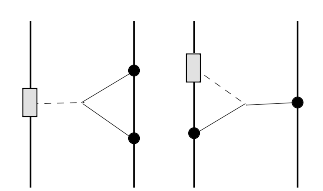

(a6)

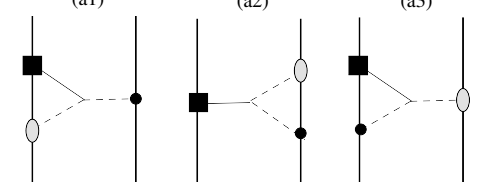

(b1)

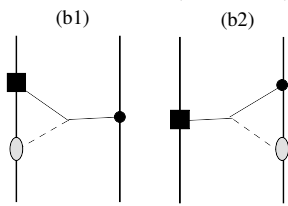

(c1)

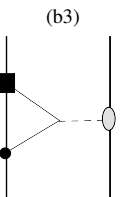

(c3) (a4)

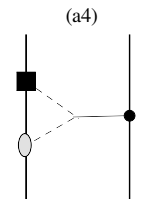

(b4)

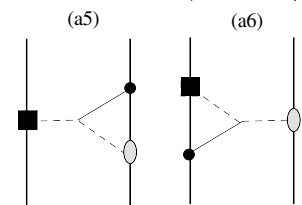

(b5) (a7)

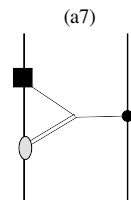

(b7)

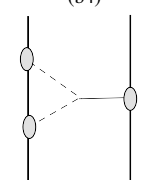

(c4)

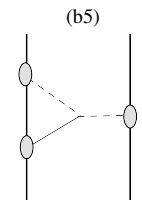

(c5)

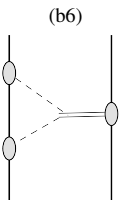

(c6)

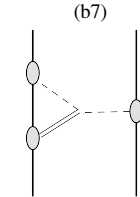

(c7)

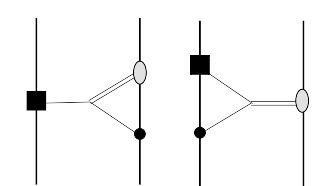

(b8)

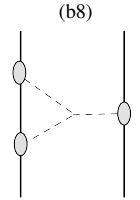

(c8)

Figure 4. The Feynman graphs at one-loop level, i.e. with cubic self-gravitational interaction, which contribute to the NLO cubic-in-spin interaction at the $4.5 \mathrm{PN}$ order for maximally-rotating compact objects. The graphs should be included together with their mirror images, i.e. with the worldline labels $1 \leftrightarrow 2$ exchanged. Similar to the nonlinear graphs of two-graviton exchange, these graphs include all relevant interactions among the spin-induced quadrupole, octupole, and the mass and spin, and we have here nonlinearities originating from gravitons sourced strictly from minimal coupling to the worldline, as shown in graphs (c4)-(c8). We also have here cubic vertices containing time derivatives, similar to what we have in the NLO odd-parity spin-orbit sector $[8,12,13]$.

Fig. $3(\mathrm{~b} 12)=-12 C_{1\left(E S^{2}\right)} \frac{G^{2}}{r^{5}}\left[2 \vec{S}_{1} \cdot \vec{S}_{2} \times \vec{v}_{1} \vec{S}_{1} \cdot \vec{n}+\vec{S}_{2} \cdot \vec{v}_{1} \times \vec{n}\left(S_{1}^{2}-5\left(\vec{S}_{1} \cdot \vec{n}\right)^{2}\right)\right]$

$$
+12 C_{1\left(E S^{2}\right)} \frac{G^{2}}{r^{4}}\left[\vec{S}_{1} \cdot \vec{S}_{2} \times \vec{n} \dot{\vec{S}}_{1} \cdot \vec{n}+\dot{\vec{S}}_{1} \cdot \vec{S}_{2} \times \vec{n} \vec{S}_{1} \cdot \vec{n}\right]
$$

Fig. $3(\mathrm{~b} 13)=2 \frac{G^{2}}{r^{5}}\left[\vec{S}_{1} \cdot \vec{v}_{2} \times \vec{n}\left(\vec{S}_{1} \cdot \vec{S}_{2}-3 \vec{S}_{1} \cdot \vec{n} \vec{S}_{2} \cdot \vec{n}\right)-\vec{S}_{1} \cdot \vec{S}_{2} \vec{S}_{1} \cdot \vec{v}_{1} \times \vec{n}\right]$

$$
+2 \frac{G^{2}}{r^{4}}\left[\dot{\vec{S}}_{1} \cdot \vec{n} \vec{S}_{1} \cdot \vec{S}_{2} \times \vec{n}-\vec{S}_{1} \cdot \vec{n} \dot{\vec{S}}_{1} \cdot \vec{S}_{2} \times \vec{n}-\dot{\vec{S}}_{1} \cdot \vec{S}_{1} \times \vec{S}_{2}\right]
$$

Fig. 3(b14) $=-8 \frac{G^{2}}{r^{5}} \vec{S}_{1} \cdot \vec{v}_{1} \times \vec{n}\left[\vec{S}_{1} \cdot \vec{S}_{2}-3 \vec{S}_{1} \cdot \vec{n} \vec{S}_{2} \cdot \vec{n}\right]$,

Fig. $3(\mathrm{~b} 15)=-\frac{G^{2}}{r^{5}}\left[2 \vec{S}_{1} \cdot \vec{S}_{2} \times \vec{n} \vec{S}_{1} \cdot \vec{v}_{1}-\vec{S}_{1} \cdot \vec{v}_{1} \times \vec{n}\left(5 \vec{S}_{1} \cdot \vec{S}_{2}-9 \vec{S}_{1} \cdot \vec{n} \vec{S}_{2} \cdot \vec{n}\right)\right.$

$$
\left.+3 \vec{S}_{2} \cdot \vec{v}_{1} \times \vec{n}\left(S_{1}^{2}-\left(\vec{S}_{1} \cdot \vec{n}\right)^{2}\right)\right] .
$$

\subsection{Cubic self-interaction}

As can be seen in figure 4 we have 24 graphs of cubic self-interaction in this sector, 6 of which contain time-dependent self-interaction, similar to what we have in the odd-parity spin-orbit sector $[8,12,13]$. Similar to the nonlinear graphs of two-graviton exchange, these graphs include all relevant interactions among the spin-induced quadrupole, octupole, and the mass and spin, and we have here nonlinearities originating from gravitons sourced strictly from minimal coupling to the worldline, as shown in graphs (c4)-(c8). This sector required using tensor one-loop integrals of up to order 5 . 
The graphs in figure 4 are evaluated as follows:

Fig. $4(\mathrm{a} 1)=-\frac{16}{3} C_{1\left(B S^{3}\right)} \frac{G^{2}}{r^{5}} \frac{m_{2}^{2}}{m_{1}^{2}} \vec{S}_{1} \cdot \vec{v}_{2} \times \vec{n}\left[S_{1}^{2}-6\left(\vec{S}_{1} \cdot \vec{n}\right)^{2}\right]$,

Fig. $4(\mathrm{a} 2)=-\frac{3}{2} C_{1\left(B S^{3}\right)} \frac{G^{2}}{r^{5}} \frac{m_{2}}{m_{1}} \vec{S}_{1} \cdot \vec{v}_{2} \times \vec{n}\left[S_{1}^{2}-5\left(\vec{S}_{1} \cdot \vec{n}\right)^{2}\right]$,

Fig. $4(\mathrm{a} 3)=\frac{3}{2} C_{1\left(B S^{3}\right)} \frac{G^{2}}{r^{5}} \frac{m_{2}}{m_{1}} \vec{S}_{1} \cdot \vec{v}_{1} \times \vec{n}\left[S_{1}^{2}-5\left(\vec{S}_{1} \cdot \vec{n}\right)^{2}\right]$,

Fig. $4(\mathrm{a} 4)=-\frac{1}{3} C_{1\left(B S^{3}\right)} \frac{G^{2}}{r^{5}} \frac{m_{2}^{2}}{m_{1}^{2}} \vec{S}_{1} \cdot \vec{v}_{1} \times \vec{n}\left[S_{1}^{2}-6\left(\vec{S}_{1} \cdot \vec{n}\right)^{2}\right]$,

Fig. $\left.4(\mathrm{a} 5)=-\frac{1}{8} C_{1\left(B S^{3}\right)} \frac{G^{2}}{r^{5}} \frac{m_{2}}{m_{1}} \vec{S}_{1} \cdot \vec{v}_{1} \times \vec{n}\left[S_{1}^{2}-5\left(\vec{S}_{1} \cdot \vec{n}\right)^{2}\right)\right]$,

Fig. $4(\mathrm{a} 6)=\frac{1}{3} C_{1\left(B S^{3}\right)} \frac{G^{2}}{r^{5}} \frac{m_{2}^{2}}{m_{1}^{2}} \vec{S}_{1} \cdot \vec{v}_{2} \times \vec{n}\left[S_{1}^{2}-6\left(\vec{S}_{1} \cdot \vec{n}\right)^{2}\right]$,

Fig. $4(\mathrm{a} 7)=\frac{1}{8} C_{1\left(B S^{3}\right)} \frac{G^{2}}{r^{5}} \frac{m_{2}}{m_{1}} \vec{S}_{1} \cdot \vec{v}_{1} \times \vec{n}\left[S_{1}^{2}-5\left(\vec{S}_{1} \cdot \vec{n}\right)^{2}\right]$,

Fig. $4(\mathrm{~b} 1)=-\frac{1}{2} C_{1\left(E S^{2}\right)} \frac{G^{2}}{r^{5}} \frac{m_{2}}{m_{1}} \vec{S}_{1} \cdot \vec{v}_{2} \times \vec{n}\left[S_{1}^{2}+3\left(\vec{S}_{1} \cdot \vec{n}\right)^{2}\right]$,

Fig. $4(\mathrm{~b} 2)=-8 C_{1\left(E S^{2}\right)} \frac{G^{2}}{r^{5}} \frac{m_{2}}{m_{1}}\left[\vec{S}_{1} \cdot \vec{S}_{2} \times \vec{v}_{2} \vec{S}_{1} \cdot \vec{n}+\vec{S}_{2} \cdot \vec{v}_{2} \times \vec{n}\left(S_{1}^{2}-3\left(\vec{S}_{1} \cdot \vec{n}\right)^{2}\right)\right]$,

Fig. $4(\mathrm{~b} 3)=4 C_{1\left(E S^{2}\right)} \frac{G^{2}}{r^{5}}\left[4 \vec{S}_{1} \cdot \vec{S}_{2} \times \vec{v}_{1} \vec{S}_{1} \cdot \vec{n}+\vec{S}_{1} \cdot \vec{S}_{2} \times \vec{n}\left(\vec{S}_{1} \cdot \vec{v}_{1}-6 \vec{S}_{1} \cdot \vec{n} \vec{v}_{1} \cdot \vec{n}\right)\right.$

$$
\left.+\vec{S}_{2} \cdot \vec{v}_{1} \times \vec{n}\left(2 S_{1}^{2}-9\left(\vec{S}_{1} \cdot \vec{n}\right)^{2}\right)\right],
$$

Fig. $4(\mathrm{~b} 4)=\frac{1}{2} C_{1\left(E S^{2}\right)} \frac{G^{2}}{r^{5}} \frac{m_{2}}{m_{1}} \vec{S}_{1} \cdot \vec{v}_{1} \times \vec{n}\left[S_{1}^{2}+3\left(\vec{S}_{1} \cdot \vec{n}\right)^{2}\right]$

$$
-2 C_{1\left(E S^{2}\right)} \frac{G^{2}}{r^{4}} \frac{m_{2}}{m_{1}} \dot{\vec{S}}_{1} \cdot \vec{S}_{1} \times \vec{n} \vec{S}_{1} \cdot \vec{n}
$$

Fig. $4(\mathrm{~b} 5)=8 C_{1\left(E S^{2}\right)} \frac{G^{2}}{r^{5}} \frac{m_{2}}{m_{1}}\left[\vec{S}_{1} \cdot \vec{S}_{2} \times \vec{v}_{1} \vec{S}_{1} \cdot \vec{n}+\vec{S}_{2} \cdot \vec{v}_{1} \times \vec{n}\left(S_{1}^{2}-3\left(\vec{S}_{1} \cdot \vec{n}\right)^{2}\right)\right]$

$$
-4 C_{1\left(E S^{2}\right)} \frac{G^{2}}{r^{4}} \frac{m_{2}}{m_{1}}\left[\vec{S}_{1} \cdot \vec{S}_{2} \times \vec{n} \dot{\vec{S}}_{1} \cdot \vec{n}+\dot{\vec{S}}_{1} \cdot \vec{S}_{2} \times \vec{n} \vec{S}_{1} \cdot \vec{n}\right],
$$

Fig. $4(\mathrm{~b} 6)=4 C_{1\left(E S^{2}\right)} \frac{G^{2}}{r^{5}}\left[2 \vec{S}_{1} \cdot \vec{S}_{2} \times \vec{v}_{1} \vec{S}_{1} \cdot \vec{n}-\vec{S}_{1} \cdot \vec{S}_{2} \times \vec{n}\left(\vec{S}_{1} \cdot \vec{v}_{1}-6 \vec{S}_{1} \cdot \vec{n} \vec{v} \cdot \vec{n}\right)\right.$

$$
\begin{aligned}
& \left.+\vec{S}_{2} \cdot \vec{v}_{1} \times \vec{n}\left(2 S_{1}^{2}-9\left(\vec{S}_{1} \cdot \vec{n}\right)^{2}\right)\right] \\
& -12 C_{1\left(E S^{2}\right)} \frac{G^{2}}{r^{4}}\left[\vec{S}_{1} \cdot \vec{S}_{2} \times \vec{n} \dot{\vec{S}}_{1} \cdot \vec{n}+\dot{\vec{S}}_{1} \cdot \vec{S}_{2} \times \vec{n} \vec{S}_{1} \cdot \vec{n}\right],
\end{aligned}
$$

Fig. $4(\mathrm{~b} 7)=-\frac{3}{8} C_{1\left(E S^{2}\right)} \frac{G^{2}}{r^{5}} \frac{m_{2}}{m_{1}} \vec{S}_{1} \cdot \vec{v}_{1} \times \vec{n}\left[S_{1}^{2}-5\left(\vec{S}_{1} \cdot \vec{n}\right)^{2}\right]$,

Fig. $4(\mathrm{~b} 8)=2 C_{1\left(E S^{2}\right)} \frac{G^{2}}{r^{5}} \frac{m_{2}}{m_{1}}\left[\vec{S}_{1} \cdot \vec{S}_{2} \times \vec{v}_{2} \vec{S}_{1} \cdot \vec{n}+\vec{S}_{2} \cdot \vec{v}_{2} \times \vec{n}\left(S_{1}^{2}-3\left(\vec{S}_{1} \cdot \vec{n}\right)^{2}\right)\right]$,

Fig. $4\left(\right.$ b9) $=\frac{1}{4} C_{1\left(E S^{2}\right)} \frac{G^{2}}{r^{5}}\left[4 \vec{S}_{1} \cdot \vec{S}_{2} \times \vec{v}_{2} \vec{S}_{1} \cdot \vec{n}-2 \vec{S}_{1} \cdot \vec{S}_{2} \times \vec{n}\left(\vec{S}_{1} \cdot \vec{v}_{2}-3 \vec{S}_{1} \cdot \vec{n} \vec{v}_{2} \cdot \vec{n}\right)\right.$

$$
\left.+3 \vec{S}_{2} \cdot \vec{v}_{2} \times \vec{n}\left(S_{1}^{2}-5\left(\vec{S}_{1} \cdot \vec{n}\right)^{2}\right)\right]
$$


Fig. $4(\mathrm{c} 1)=\frac{3}{8} C_{1\left(E S^{2}\right)} \frac{G^{2}}{r^{5}} \frac{m_{2}}{m_{1}}\left[\vec{S}_{1} \cdot \vec{v}_{1} \times \vec{n}\left(S_{1}^{2}-5\left(\vec{S}_{1} \cdot \vec{n}\right)^{2}\right)\right]$

$$
-C_{1\left(E S^{2}\right)} \frac{G^{2}}{r^{4}} \frac{m_{2}}{m_{1}}\left[\dot{\vec{S}}_{1} \cdot \vec{S}_{1} \times \vec{n} \vec{S}_{1} \cdot \vec{n}\right]
$$

Fig. $4(\mathrm{c} 2)=-2 C_{1\left(E S^{2}\right)} \frac{G^{2}}{r^{5}} \frac{m_{2}}{m_{1}}\left[\vec{S}_{1} \cdot \vec{S}_{2} \times \vec{v}_{2} \vec{S}_{1} \cdot \vec{n}+\vec{S}_{2} \cdot \vec{v}_{2} \times \vec{n}\left(S_{1}^{2}-3\left(\vec{S}_{1} \cdot \vec{n}\right)^{2}\right)\right]$,

Fig. $4(\mathrm{c} 3)=-\frac{1}{4} C_{1\left(E S^{2}\right)} \frac{G^{2}}{r^{5}}\left[4 \vec{S}_{1} \cdot \vec{S}_{2} \times \vec{v}_{1} \vec{S}_{1} \cdot \vec{n}-2 \vec{S}_{1} \cdot \vec{S}_{2} \times \vec{n}\left(\vec{S}_{1} \cdot \vec{v}_{1}-3 \vec{S}_{1} \cdot \vec{n} \vec{v}_{1} \cdot \vec{n}\right)\right.$

$$
\begin{aligned}
& \left.+3 \vec{S}_{2} \cdot \vec{v}_{1} \times \vec{n}\left(S_{1}^{2}-5\left(\vec{S}_{1} \cdot \vec{n}\right)^{2}\right)\right] \\
& +C_{1\left(E S^{2}\right)} \frac{G^{2}}{r^{4}}\left[\vec{S}_{1} \cdot \vec{S}_{2} \times \vec{n} \dot{\vec{S}}_{1} \cdot \vec{n}+\dot{\vec{S}}_{1} \cdot \vec{S}_{2} \times \vec{n} \vec{S}_{1} \cdot \vec{n}\right],
\end{aligned}
$$

Fig. $4(\mathrm{c} 4)=4 \frac{G^{2}}{r^{5}}\left[\vec{S}_{1} \cdot \vec{S}_{2} \times \vec{v}_{2} \vec{S}_{1} \cdot \vec{n}-3 \vec{S}_{2} \cdot \vec{v}_{2} \times \vec{n}\left(\vec{S}_{1} \cdot \vec{n}\right)^{2}\right]$,

Fig. $4(\mathrm{c} 5)=4 \frac{G^{2}}{r^{5}}\left[\vec{S}_{1} \cdot \vec{S}_{2} \times \vec{v}_{1} \vec{S}_{1} \cdot \vec{n}+\vec{S}_{1} \cdot \vec{v}_{1} \times \vec{n} \vec{S}_{1} \cdot \vec{S}_{2}\right]$,

Fig. $4(\mathrm{c} 6)=-\frac{1}{2} \frac{G^{2}}{r^{5}}\left[15 \vec{S}_{1} \cdot \vec{S}_{2} \times \vec{n}\left(\vec{S}_{1} \cdot \vec{v}_{2}-\vec{S}_{1} \cdot \vec{n} \vec{v}_{2} \cdot \vec{n}\right)\right.$

$$
\begin{aligned}
& -\vec{S}_{1} \cdot \vec{v}_{2} \times \vec{n}\left(14 \vec{S}_{1} \cdot \vec{S}_{2}-12 \vec{S}_{1} \cdot \vec{n} \vec{S}_{2} \cdot \vec{n}\right) \\
& \left.+\frac{1}{2} \vec{S}_{2} \cdot \vec{v}_{2} \times \vec{n}\left(29 S_{1}^{2}-33\left(\vec{S}_{1} \cdot \vec{n}\right)^{2}\right)\right],
\end{aligned}
$$

Fig. $4(\mathrm{c} 7)=-\frac{G^{2}}{r^{5}}\left[4 \vec{S}_{1} \cdot \vec{S}_{2} \times \vec{v}_{1} \vec{S}_{1} \cdot \vec{n}+3 \vec{S}_{1} \cdot \vec{S}_{2} \times \vec{n}\left(\vec{S}_{1} \cdot \vec{v}_{1}-4 \vec{S}_{1} \cdot \vec{n} \vec{v}_{1} \cdot \vec{n}\right)\right.$

$$
\left.+\vec{S}_{2} \cdot \vec{v}_{1} \times \vec{n}\left(S_{1}^{2}-6\left(\vec{S}_{1} \cdot \vec{n}\right)^{2}\right)\right]
$$

Fig. $4(\mathrm{c} 8)=\frac{1}{4} \frac{G^{2}}{r^{5}}\left[4 \overrightarrow{S_{1}} \cdot \vec{S}_{2} \times \vec{v}_{1} \vec{S}_{1} \cdot \vec{n}+8 \overrightarrow{S_{1}} \cdot \vec{S}_{2} \times \vec{v}_{2} \vec{S}_{1} \cdot \vec{n}\right.$

$$
\begin{aligned}
& +6 \vec{S}_{1} \cdot \vec{S}_{2} \times \vec{n}\left(\vec{S}_{1} \cdot \vec{v}_{1}-5 \vec{S}_{1} \cdot \vec{n} \vec{v}_{1} \cdot \vec{n}\right)+8 \vec{S}_{1} \cdot \vec{S}_{2} \times \vec{n}\left(\vec{S}_{1} \cdot \vec{v}_{2}-6 \vec{S}_{1} \cdot \vec{n} \vec{v}_{2} \cdot \vec{n}\right) \\
& \left.+8 \vec{S}_{1} \cdot \vec{v}_{1} \times \vec{n}\left(\vec{S}_{1} \cdot \vec{S}_{2}-3 \vec{S}_{1} \cdot \vec{n} \vec{S}_{2} \cdot \vec{n}\right)+\vec{S}_{2} \cdot \vec{v}_{1} \times \vec{n}\left(5 S_{1}^{2}-9\left(\vec{S}_{1} \cdot \vec{n}\right)^{2}\right)\right] \\
& +\frac{G^{2}}{r^{4}}\left[\vec{S}_{1} \cdot \vec{S}_{2} \times \vec{n} \dot{\vec{S}}_{1} \cdot \vec{n}-2 \vec{S}_{1} \cdot \dot{\vec{S}}_{2} \times \vec{n} \vec{S}_{1} \cdot \vec{n}+\dot{\vec{S}}_{1} \cdot \vec{S}_{2} \times \vec{n} \vec{S}_{1} \cdot \vec{n}\right] .
\end{aligned}
$$

\section{New features from spin dependence of linear momentum}

The formulation of the EFT of a spinning gravitating particle in [12] consisted of an action initially taken in the covariant gauge as introduced by Tulczyjew in [48] (later extended to higher-multipoles by Dixon [49]). Tulczyjew put forward the spin supplementary condition (SSC) given by $S_{\mu \nu} p^{\nu}=0$, which as noted in [12], corresponds to the choice $e_{0}^{\mu}=p^{\mu} / \sqrt{p^{2}}$ for the timelike component of the worldline tetrad in terms of the linear momentum $p^{\mu}$. This gauge is distinguished among possible covariant gauges, in particular with the fourvelocity $u^{\mu}$ (as in $S_{\mu \nu} u^{\nu}=0$ ) as the only gauge of rotational DOFs for which the existence and uniqueness of a corresponding "center" for the spinning particle were proven rigorously in General Relativity [50, 51]. 
For this reason the formulation in [12] was made in terms of the linear momentum $p^{\mu}$, rather than the four-velocity $u^{\mu}$, e.g., as in general the former is given by

$$
p_{\mu}=-\frac{\partial L}{\partial u^{\mu}}=m \frac{u_{\mu}}{\sqrt{u}^{2}}+\mathcal{O}\left(R S^{2}\right)
$$

where we recall that the Lagrangian is first constructed with the 'spin gauge-invariant' variable, as explained in [12]. Therefore the spin-dependent difference between $p_{\mu}$ and $u_{\mu}$ would show up, as was pointed out in [12], as of the NLO of the sector cubic in the spins, namely the sector that we are studying in this work.

Let us then find how this new feature transpires in this sector. Since we are working to cubic order in the spin in this sector, we should take into account in the linear momentum beyond the leading term only the first correction, that is we now consider also

$$
\Delta p_{\kappa}[S] \equiv p_{\kappa}-\bar{p}_{\kappa} \simeq \frac{C_{E S^{2}}}{2 m} S^{\mu} S^{\nu}\left(\frac{2}{u} R_{\mu \alpha \nu \kappa} u^{\alpha}-\frac{1}{u^{3}} R_{\mu \alpha \nu \beta} u^{\alpha} u^{\beta} u_{\kappa}\right),
$$

where we denoted the leading approximation to the linear momentum as $\bar{p}_{\kappa} \equiv \frac{m}{u} u_{\kappa}$. Let us also note that due to eq. (4.8) of [12] at this order the expression with spin vectors can be used interchangeably as that with the spin tensors. The appearance of $u^{\mu}$ in $p^{\mu}$ itself thus requires further inquiry only as of the NLO quartic-in-spin sector [20], where it was in fact found that this subtlety is still irrelevant until even higher PN orders.

Hence, the part that is linear in the spin in the action of the spinning particle actually gives rise to a new type of worldline-graviton couplings that are cubic in the spin, due to its dependence in the linear momentum. We recall that the relevant part of the Lagrangian is given as follows [12]:

$$
L_{S}=-\frac{1}{2} \hat{S}_{a b} \hat{\Omega}_{\text {flat }}^{a b}-\frac{1}{2} \hat{S}_{a b} \omega_{\mu}^{a b} u^{\mu}-\frac{\hat{S}_{a b} p^{b}}{p^{2}} \frac{D p^{a}}{D \sigma},
$$

where the hatted DOFs represent the generic rotational DOFs. Therefore the new contributions arise from substituting in the linear-in-spin couplings the gauge, which we choose here as the canonical gauge, formulated in [12] as

$$
\hat{\Lambda}_{[0]}^{a}=\delta_{0}^{a}, \quad \hat{S}^{a b}\left(p_{b}+p \delta_{0 b}\right)=0,
$$

as well as from the extra term that enters from minimal coupling, appearing last in eq. (4.3), which was found in [12] to be related with the gauge of the rotational DOFs, and stands for the Thomas precession as noted in section 2. Let us stress again that the subtlety here is not about switching from the covariant gauge, but rather about advancing from using $u_{\nu}$ in the basic covariant gauge, to using in it the spin-dependent $p_{\nu}$, which is necessary as of this cubic order in spins and nonlinear order in gravity.

Working out explicitly this part of the action in terms of the local spin variable in the canonical gauge similarly to the derivations in [12], and keeping only terms that lead to new cubic-in-spin terms, we obtain here the following contribution:

$$
L_{S \rightarrow S^{3}}=\omega_{\mu}^{i j} u^{\mu} \frac{\hat{S}_{i k} p^{k} p_{j}}{p\left(p+p^{0}\right)}-\omega_{\mu}^{0 i} u^{\mu} \frac{\hat{S}_{i j} p^{j}}{p}+\frac{\hat{S}_{i j} p^{i} \dot{p}^{j}}{p\left(p+p^{0}\right)},
$$


where in principle all the temporal and spatial indices that are specified here are in the locally flat frame. In order to obtain the new cubic-in-spin couplings we only need to substitute in the correction to the linear momentum from eq. (4.2) to linear order, keeping in mind that all of the contributions at the zeroth order are taken into account in the Feynman rules from past sectors, e.g. [12,13], and from section 2 above. At this point it becomes clear that the first two terms in eq. (4.5) give rise to new two-graviton couplings, and that the last term gives rise to new one-graviton couplings containing higher-order time derivatives.

The resulting new Feynman rules for the one-graviton couplings are then:

$$
\begin{aligned}
& \}_{---}=\int d t\left[\frac{C_{\mathrm{ES}^{2}}}{4 m^{2}} S_{i} S_{j} \epsilon_{k l m}\left[\left(2 S_{m} a^{k}+\dot{S}_{m} v^{k}\right)\left(A_{l, i j}-A_{j, i l}\right)\right]\right], \\
& \hat{Q}=\int d t\left[-\frac{C_{\mathrm{ES}^{2}}}{2 m^{2}} S_{i} S_{j} \epsilon_{k l m}\left[2 S_{m} a^{k}\left(2\left(\phi_{, i j} v^{l}-\phi_{, i l} v^{j}\right)+\delta_{i j}\left(\partial_{t} \phi_{, l}+\phi_{, l n} v^{n}\right)\right)\right.\right. \\
& \left.\left.-\dot{S}_{m} v^{k}\left(2 \phi_{, i l} v^{j}-\delta_{i j}\left(\partial_{t} \phi_{, l}+\phi_{, l n} v^{n}\right)+\delta_{i l}\left(\partial_{t} \phi_{, j}+\phi_{, j n} v^{n}\right)\right)\right]\right],
\end{aligned}
$$

where a black square mounted on a gray oval blob represents this new type of "composite" cubic-in-spin worldline couplings. Notice that all these rules contain accelerations and even time derivatives of spins, similar to the acceleration terms that appear first in the rules for the spin-orbit sector [12]. Note also that at this level the new couplings depend linearly on a single Wilson coefficient.

For the new two-graviton couplings we get the following rules:

$$
\begin{aligned}
& =\int d t\left[\frac{C_{\mathrm{ES}^{2}}}{2 m^{2}} S_{i} S_{j} \epsilon_{k l m} S_{m} \phi_{, k}\left(A_{l, i j}-A_{j, i l}\right)\right], \\
& =\int d t\left[-\frac{C_{\mathrm{ES}}}{m^{2}} S_{i} S_{j} \epsilon_{k l m} S_{m} \phi_{, k}\left(2\left(\phi_{, i j} v^{l}-\phi_{, i l} v^{j}\right)+\delta_{i j}\left(\partial_{t} \phi_{, l}+\phi_{, l n} v^{n}\right)\right)\right] .
\end{aligned}
$$

Note that the mass ratio together with the Wilson coefficient in these new rules for cubicin-spin couplings indicate that these are truly new couplings that cannot be absorbed in the existing "elementary" octupole operator.

These new couplings give rise to 4 additional graphs as shown in figure 5, similar to those in figure 2 (a1), (a2), and in figure 3 (b1), (b2). The graphs in figure 5 are evaluated as follows:

$$
\begin{aligned}
\text { Fig. 5(a1)= } & -C_{1\left(E S^{2}\right)} \frac{G}{r^{3}} \frac{m_{2}}{m_{1}^{2}}\left[2 \vec{S}_{1} \cdot \vec{v}_{2} \times \vec{a}_{1}\left(S_{1}^{2}-3\left(\vec{S}_{1} \cdot \vec{n}\right)^{2}\right)-6 \vec{S}_{1} \cdot \vec{a}_{1} \times \vec{n}_{S_{1}} \cdot \vec{v}_{2} \vec{S}_{1} \cdot \vec{n}\right. \\
& -\dot{\vec{S}}_{1} \cdot \vec{S}_{1} \times \vec{v}_{1} \vec{S}_{1} \cdot \vec{v}_{2}-\dot{\vec{S}}_{1} \cdot \vec{v}_{1} \times \vec{v}_{2}\left(S_{1}^{2}-3\left(\vec{S}_{1} \cdot \vec{n}\right)^{2}\right) \\
& \left.-3 \dot{\vec{S}}_{1} \cdot \vec{v}_{1} \times \vec{n} \vec{S}_{1} \cdot \vec{v}_{2} \vec{S}_{1} \cdot \vec{n}\right]
\end{aligned}
$$




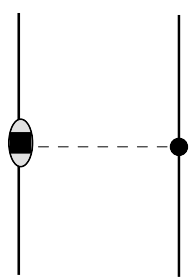

(a1)

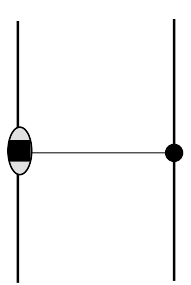

(a2)

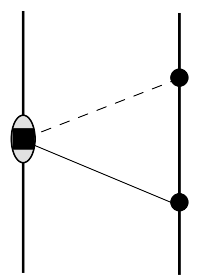

(b1)

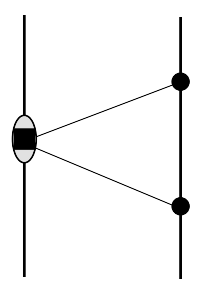

(b2)

Figure 5. The extra Feynman graphs of one- and two-graviton exchange, which appear at the NLO cubic-in-spin interaction at the $4.5 \mathrm{PN}$ order for maximally-rotating compact objects. The graphs should be included together with their mirror images, i.e. with the worldline labels $1 \leftrightarrow 2$ exchanged. These graphs contain a new type of worldline-graviton couplings, which we refer to as "composite" octupole ones, and obviously yield similar graphs to the corresponding ones with the "elementary" spin-induced octupole couplings in figure 2(a1),(a2) and in figure 3(b1),(b2).

Fig. $5(\mathrm{a} 2)=\frac{1}{2} C_{1\left(E S^{2}\right)} \frac{G}{r^{3}} \frac{m_{2}}{m_{1}^{2}}\left[6 \vec{S}_{1} \cdot \vec{v}_{1} \times \vec{a}_{1}\left(S_{1}^{2}-2\left(\vec{S}_{1} \cdot \vec{n}\right)^{2}\right)-2 \vec{S}_{1} \cdot \vec{v}_{2} \times \vec{a}_{1} S_{1}^{2}\right.$

$$
\begin{aligned}
& +6 \vec{S}_{1} \cdot \vec{a}_{1} \times \vec{n}\left(S_{1}^{2}\left(\vec{v}_{1} \cdot \vec{n}-\vec{v}_{2} \cdot \vec{n}\right)-2 \vec{S}_{1} \cdot \vec{v}_{1} \vec{S}_{1} \cdot \vec{n}\right) \\
& -\dot{\vec{S}}_{1} \cdot \vec{S}_{1} \times \vec{v}_{1}\left(3 \vec{S}_{1} \cdot \vec{v}_{1}-\vec{S}_{1} \cdot \vec{v}_{2}-3 \vec{S}_{1} \cdot \vec{n}\left(\vec{v}_{1} \cdot \vec{n}-\vec{v}_{2} \cdot \vec{n}\right)\right) \\
& \left.+\dot{\vec{S}}_{1} \cdot \vec{v}_{1} \times \vec{v}_{2} S_{1}^{2}+3 \dot{\vec{S}}_{1} \cdot \vec{v}_{1} \times \vec{n}\left(S_{1}^{2}\left(\vec{v}_{1} \cdot \vec{n}-\vec{v}_{2} \cdot \vec{n}\right)-2 \vec{S}_{1} \cdot \vec{v}_{1} \vec{S}_{1} \cdot \vec{n}\right)\right]
\end{aligned}
$$

Fig. $5(\mathrm{~b} 1)=-2 C_{1\left(E S^{2}\right)} \frac{G^{2}}{r^{5}} \frac{m_{2}^{2}}{m_{1}^{2}} \vec{S}_{1} \cdot \vec{v}_{2} \times \vec{n}\left[S_{1}^{2}-3\left(\vec{S}_{1} \cdot \vec{n}\right)^{2}\right]$,

Fig. $5(\mathrm{~b} 2)=C_{1\left(E S^{2}\right)} \frac{G^{2}}{r^{5}} \frac{m_{2}^{2}}{m_{1}^{2}}\left[3 \vec{S}_{1} \cdot \vec{v}_{1} \times \vec{n}\left(S_{1}^{2}-2\left(\vec{S}_{1} \cdot \vec{n}\right)^{2}\right)-\vec{S}_{1} \cdot \vec{v}_{2} \times \vec{n} S_{1}^{2}\right]$.

\section{The gravitational cubic-in-spin action at the next-to-leading order}

Let us then put together all the results from sections 3 and 4 to get the final effective action for this sector. This summation includes the values presented above plus similar results under the exchange of particle labels $1 \leftrightarrow 2$, where $\vec{n} \rightarrow-\vec{n}$. Next, we apply the 4 -vectors identity for 3 dimensions presented in eq. (3.14) of [11], to further simplify and compress the results. As was already noted these results contain higher-order time derivatives of both the velocity and the spin, which will be treated rigorously at the level of the action, following the procedure shown in [47], by making variable redefinitions that will remove the higher-order terms (in complete analogy to the removal of redundant/on-shell operators by field redefinitions in effective field theories, as was pointed out by one of the authors in [47]).

The final result of these steps is then given as follows:

$$
L_{\mathrm{S}^{3}}^{\mathrm{NLO}}=L_{\mathrm{S}_{1}^{2} \mathrm{~S}_{2}}^{\mathrm{NLO}}+L_{\mathrm{S}_{1}^{3}}^{\mathrm{NLO}}+(1 \leftrightarrow 2),
$$

where we have:

$$
L_{\mathrm{S}_{1}^{2} \mathrm{~S}_{2}}^{\mathrm{NLO}}=+\frac{G^{2}}{r^{5}} L_{(1)}+C_{1\left(E S^{2}\right)} \frac{G}{r^{4}} \frac{1}{m_{1}} L_{(2)}+C_{1\left(E S^{2}\right)} \frac{G^{2}}{r^{5}} L_{(3)}+C_{1\left(E S^{2}\right)} \frac{G^{2} m_{2}}{r^{5} m_{1}} L_{(4)}
$$




$$
\begin{aligned}
& +\frac{G^{2}}{r^{4}} L_{(5)}+C_{1\left(E S^{2}\right)} \frac{G}{r^{3}} \frac{1}{m_{1}} L_{(6)}+C_{1\left(E S^{2}\right)} \frac{G^{2}}{r^{4}} L_{(7)}+C_{1\left(E S^{2}\right)} \frac{G^{2} m_{2}}{r^{4} m_{1}} L_{(8)} \\
& +C_{1\left(E S^{2}\right)} \frac{G}{r^{2}} \frac{1}{m_{1}} L_{(9)}+C_{1\left(E S^{2}\right)} \frac{G}{r} \frac{1}{m_{1}} L_{(10)},
\end{aligned}
$$

with the following pieces:

$$
\begin{aligned}
& L_{(1)}=\frac{1}{2} \vec{S}_{1} \cdot \vec{S}_{2} \times \vec{n}\left(-5 \vec{S}_{1} \cdot \vec{v}_{1}+\vec{S}_{1} \cdot \vec{v}_{2}+9 \vec{S}_{1} \cdot \vec{n} \vec{v}_{1} \cdot \vec{n}-9 \vec{S}_{1} \cdot \vec{n} \vec{v}_{2} \cdot \vec{n}\right) \\
& +9 \vec{S}_{1} \cdot \vec{v}_{1} \times \vec{n} \vec{S}_{1} \cdot \vec{n} \vec{S}_{2} \cdot \vec{n}-\frac{5}{4} \vec{S}_{2} \cdot \vec{v}_{2} \times \vec{n}\left(S_{1}^{2}+3\left(\vec{S}_{1} \cdot \vec{n}\right)^{2}\right) \\
& +\vec{S}_{1} \cdot \vec{v}_{2} \times \vec{n}\left(3 \vec{S}_{1} \cdot \vec{S}_{2}-12 \vec{S}_{1} \cdot \vec{n} \vec{S}_{2} \cdot \vec{n}\right) \\
& -\frac{1}{4} \vec{S}_{2} \cdot \vec{v}_{1} \times \vec{n}\left(7 S_{1}^{2}-27\left(\vec{S}_{1} \cdot \vec{n}\right)^{2}\right), \\
& L_{(2)}=-3 \vec{S}_{1} \cdot \vec{S}_{2} \times \vec{v}_{1}\left(4 \vec{S}_{1} \cdot \vec{v}_{1} \vec{v}_{2} \cdot \vec{n}+\vec{S}_{1} \cdot \vec{v}_{2} \vec{v}_{2} \cdot \vec{n}\right)+\frac{9}{2} \vec{S}_{2} \cdot \vec{v}_{1} \times \vec{v}_{2} S_{1}^{2} \vec{v}_{1} \cdot \vec{n} \\
& +3 \vec{S}_{1} \cdot \vec{S}_{2} \times \vec{v}_{2}\left(4 \vec{S}_{1} \cdot \vec{v}_{2} \vec{v}_{1} \cdot \vec{n}+\vec{S}_{1} \cdot \vec{n}\left(2 \vec{v}_{1} \cdot \vec{v}_{2}-v_{2}^{2}\right)\right) \\
& -3 \vec{S}_{1} \cdot \vec{v}_{1} \times \vec{v}_{2} \vec{S}_{1} \cdot \vec{S}_{2} \vec{v}_{2} \cdot \vec{n}-\frac{15}{2} \vec{v}_{1} \cdot \vec{v}_{2} \times \vec{n} \vec{S}_{2} \cdot \vec{v}_{1}\left(\vec{S}_{1} \cdot \vec{n}\right)^{2} \\
& +\vec{S}_{2} \cdot \vec{v}_{1} \times \vec{n}\left(\frac{15}{2} S_{1}^{2}\left(v_{1}^{2}-\vec{v}_{1} \cdot \vec{v}_{2}-2\left(\vec{v}_{1} \cdot \vec{n}\right)^{2}-\vec{v}_{1} \cdot \vec{n} \vec{v}_{2} \cdot \vec{n}\right)\right. \\
& +3 \vec{S}_{1} \cdot \vec{v}_{1}\left(-2 \vec{S}_{1} \cdot \vec{v}_{1}+2 \vec{S}_{1} \cdot \vec{v}_{2}+5 \vec{S}_{1} \cdot \vec{n} \vec{v}_{1} \cdot \vec{n}\right)-15 \vec{S}_{1} \cdot \vec{v}_{2} \vec{S}_{1} \cdot \vec{n} \vec{v}_{1} \cdot \vec{n} \\
& \left.+\frac{15}{2}\left(\vec{S}_{1} \cdot \vec{n}\right)^{2}\left(-v_{1}^{2}+7 \vec{v}_{1} \cdot \vec{n} \vec{v}_{2} \cdot \vec{n}\right)\right)+\vec{S}_{2} \cdot \vec{v}_{2} \times \vec{n}\left(\frac { 3 } { 2 } S _ { 1 } ^ { 2 } \left(-5 v_{1}^{2}+4 \vec{v}_{1} \cdot \vec{v}_{2}-v_{2}^{2}\right.\right. \\
& \left.+10\left(\vec{v}_{1} \cdot \vec{n}\right)^{2}+5 \vec{v}_{1} \cdot \vec{n} \vec{v}_{2} \cdot \vec{n}\right)+\frac{15}{2}\left(\vec{S}_{1} \cdot \vec{n}\right)^{2}\left(2 v_{1}^{2}-2 \vec{v}_{1} \cdot \vec{v}_{2}+v_{2}^{2}-7 \vec{v}_{1} \cdot \vec{n} \vec{v}_{2} \cdot \vec{n}\right) \\
& \left.+3 \vec{S}_{1} \cdot \vec{v}_{1}\left(2 \vec{S}_{1} \cdot \vec{v}_{1}-\vec{S}_{1} \cdot \vec{v}_{2}-5 \vec{S}_{1} \cdot \vec{n} \vec{v}_{1} \cdot \vec{n}+5 \vec{S}_{1} \cdot \vec{n} \vec{v}_{2} \cdot \vec{n}\right)\right) \\
& -3 \vec{S}_{1} \cdot \vec{v}_{1} \times \vec{n}\left(\vec{S}_{1} \cdot \vec{S}_{2}\left(v_{1}^{2}-\vec{v}_{1} \cdot \vec{v}_{2}-5 \vec{v}_{1} \cdot \vec{n} \vec{v}_{2} \cdot \vec{n}\right)+\vec{S}_{1} \cdot \vec{v}_{2} \vec{S}_{2} \cdot \vec{v}_{1}\right. \\
& \left.+\vec{S}_{1} \cdot \vec{v}_{1}\left(-\vec{S}_{2} \cdot \vec{v}_{1}+5 \vec{S}_{2} \cdot \vec{n} \vec{v}_{2} \cdot \vec{n}\right)\right)+3 \vec{S}_{1} \cdot \vec{v}_{2} \times \vec{n}\left(\vec{S}_{1} \cdot \vec{S}_{2}\left(v_{1}^{2}-5 \vec{v}_{1} \cdot \vec{n} \vec{v}_{2} \cdot \vec{n}\right)\right. \\
& \left.-\vec{S}_{1} \cdot \vec{v}_{1} \vec{S}_{2} \cdot \vec{v}_{1}+15 \vec{S}_{1} \cdot \vec{v}_{2} \vec{S}_{2} \cdot \vec{n} \vec{v}_{1} \cdot \vec{n}\right) \\
& L_{(3)}=\frac{1}{2} \vec{S}_{1} \cdot \vec{S}_{2} \times \vec{v}_{1} \vec{S}_{1} \cdot \vec{n}-\frac{1}{2} \vec{S}_{1} \cdot \vec{S}_{2} \times \vec{v}_{2} \vec{S}_{1} \cdot \vec{n}+\frac{3}{2} \vec{S}_{1} \cdot \vec{S}_{2}\left(\vec{S}_{1} \cdot \vec{v}_{1} \times \vec{n}-\vec{S}_{1} \cdot \vec{v}_{2} \times \vec{n}\right) \\
& +\vec{S}_{1} \cdot \vec{S}_{2} \times \vec{n}\left(-\vec{S}_{1} \cdot \vec{v}_{1}+\vec{S}_{1} \cdot \vec{v}_{2}-\frac{3}{2} \vec{S}_{1} \cdot \vec{n} \vec{v}_{1} \cdot \vec{n}+\frac{3}{2} \vec{S}_{1} \cdot \vec{n} \vec{v}_{2} \cdot \vec{n}\right) \\
& -\frac{3}{4}\left(\vec{S}_{2} \cdot \vec{v}_{1} \times \vec{n}-\vec{S}_{2} \cdot \vec{v}_{2} \times \vec{n}\right)\left(3 S_{1}^{2}-5\left(\vec{S}_{1} \cdot \vec{n}\right)^{2}\right), \\
& L_{(4)}=31 \vec{S}_{1} \cdot \vec{S}_{2}\left(\vec{S}_{1} \cdot \vec{v}_{1} \times \vec{n}-\vec{S}_{1} \cdot \vec{v}_{2} \times \vec{n}\right) \\
& -2\left(\vec{S}_{2} \cdot \vec{v}_{1} \times \vec{n}-\vec{S}_{2} \cdot \vec{v}_{2} \times \vec{n}\right)\left(19 S_{1}^{2}-21\left(\vec{S}_{1} \cdot \vec{n}\right)^{2}\right) \\
& +\vec{S}_{1} \cdot \vec{S}_{2} \times \vec{n}\left(-41 \vec{S}_{1} \cdot \vec{v}_{1}+41 \vec{S}_{1} \cdot \vec{v}_{2}+63 \vec{S}_{1} \cdot \vec{n} \vec{v}_{1} \cdot \vec{n}-66 \vec{S}_{1} \cdot \vec{n} \vec{v}_{2} \cdot \vec{n}\right), \quad \text { (5.6) } \\
& L_{(5)}=3 \vec{S}_{1} \cdot \vec{S}_{2} \times \vec{n} \dot{\vec{S}}_{1} \cdot \vec{n}-\dot{\vec{S}}_{1} \cdot \vec{S}_{2} \times \vec{n} \vec{S}_{1} \cdot \vec{n}-2 \vec{S}_{1} \cdot \dot{\vec{S}}_{2} \times \vec{n} \vec{S}_{1} \cdot \vec{n}+2 \vec{S}_{1} \cdot \dot{\vec{S}}_{1} \times \vec{S}_{2},
\end{aligned}
$$




$$
\begin{aligned}
& L_{(6)}=\frac{1}{2} \vec{S}_{1} \cdot \vec{S}_{2} \times \vec{v}_{1} \dot{\vec{S}}_{1} \cdot \vec{v}_{2}+\frac{1}{2} \dot{\vec{S}}_{1} \cdot \vec{S}_{2} \times \vec{v}_{1} \vec{S}_{1} \cdot \vec{v}_{2} \\
& +\frac{1}{2} \vec{S}_{1} \cdot \vec{S}_{2} \times \vec{v}_{2}\left(\dot{\vec{S}}_{1} \cdot \vec{v}_{1}-2 \dot{\vec{S}}_{1} \cdot \vec{v}_{2}-3 \dot{\vec{S}}_{1} \cdot \vec{n} \vec{v}_{1} \cdot \vec{n}-3 \dot{\vec{S}}_{1} \cdot \vec{n} \vec{v}_{2} \cdot \vec{n}\right) \\
& +\vec{S}_{1} \cdot \vec{S}_{2} \times \vec{a}_{1} \vec{S}_{1} \cdot \vec{v}_{2}+\left(\vec{S}_{1} \cdot \vec{S}_{2} \times \vec{a}_{2}+\vec{S}_{1} \cdot \dot{\vec{S}}_{2} \times \vec{v}_{2}\right)\left(-\vec{S}_{1} \cdot \vec{v}_{1}+3 \vec{S}_{1} \cdot \vec{n} \vec{v}_{1} \cdot \vec{n}\right) \\
& -2 \vec{S}_{1} \cdot \dot{\vec{S}}_{2} \times \vec{v}_{1} \vec{S}_{1} \cdot \vec{v}_{1}+3 \vec{S}_{1} \cdot \vec{v}_{2} \times \vec{a}_{1} \vec{S}_{1} \cdot \vec{n} \vec{S}_{2} \cdot \vec{n} \\
& +\frac{1}{2} \dot{\vec{S}}_{1} \cdot \vec{S}_{2} \times \vec{v}_{2}\left(\vec{S}_{1} \cdot \vec{v}_{1}-\vec{S}_{1} \cdot \vec{v}_{2}-3 \vec{S}_{1} \cdot \vec{n} \vec{v}_{1} \cdot \vec{n}-3 \vec{S}_{1} \cdot \vec{n} \vec{v}_{2} \cdot \vec{n}\right) \\
& -\vec{S}_{2} \cdot \vec{v}_{1} \times \vec{v}_{2}\left(\dot{\vec{S}}_{1} \cdot \vec{S}_{1}-3 \dot{\vec{S}}_{1} \cdot \vec{n} \vec{S}_{1} \cdot \vec{n}\right) \\
& +\left(\vec{S}_{2} \cdot \vec{v}_{2} \times \vec{a}_{2}+\frac{3}{2} \vec{S}_{2} \cdot \vec{v}_{2} \times \vec{a}_{1}\right)\left(S_{1}^{2}-3\left(\vec{S}_{1} \cdot \vec{n}\right)^{2}\right) \\
& +\frac{3}{2} \vec{S}_{1} \cdot \vec{S}_{2} \times \vec{n}\left(2 \vec{S}_{1} \cdot \vec{n} \vec{a}_{1} \cdot \vec{v}_{2}+\dot{\vec{S}}_{1} \cdot \vec{n} \vec{v}_{1} \cdot \vec{v}_{2}-\dot{\vec{S}}_{1} \cdot \vec{v}_{2} \vec{v}_{1} \cdot \vec{n}\right. \\
& \left.+5 \dot{\vec{S}}_{1} \cdot \vec{n} \vec{v}_{1} \cdot \vec{n} \vec{v}_{2} \cdot \vec{n}\right)-\frac{1}{2}\left(\vec{S}_{2} \cdot \vec{v}_{1} \times \vec{a}_{2}+\dot{\vec{S}}_{2} \cdot \vec{v}_{1} \times \vec{v}_{2}\right)\left(5 S_{1}^{2}-9\left(\vec{S}_{1} \cdot \vec{n}\right)^{2}\right) \\
& +\frac{3}{2} \dot{\vec{S}}_{1} \cdot \vec{S}_{2} \times \vec{n}\left(-\vec{S}_{1} \cdot \vec{v}_{2} \vec{v}_{1} \cdot \vec{n}+\vec{S}_{1} \cdot \vec{n} \vec{v}_{1} \cdot \vec{v}_{2}+5 \vec{S}_{1} \cdot \vec{n} \vec{v}_{1} \cdot \vec{n} \vec{v}_{2} \cdot \vec{n}\right) \\
& +\frac{3}{2} \vec{S}_{2} \cdot \vec{v}_{1} \times \vec{n}\left(S_{1}^{2} \vec{a}_{1} \cdot \vec{n}+\dot{\vec{S}}_{1} \cdot \vec{S}_{1}\left(2 \vec{v}_{1} \cdot \vec{n}+4 \vec{v}_{2} \cdot \vec{n}\right)-10 \dot{\vec{S}}_{1} \cdot \vec{n} \vec{S}_{1} \cdot \vec{n} \vec{v}_{2} \cdot \vec{n}\right) \\
& +\frac{3}{2} \vec{S}_{2} \cdot \vec{a}_{1} \times \vec{n}\left(S_{1}^{2} \vec{v}_{1} \cdot \vec{n}+S_{1}^{2} \vec{v}_{2} \cdot \vec{n}-5\left(\overrightarrow{S_{1}} \cdot \vec{n}\right)^{2} \vec{v}_{2} \cdot \vec{n}\right) \\
& +\frac{3}{2} \vec{S}_{1} \cdot \vec{v}_{1} \times \vec{n}\left(\vec{S}_{1} \cdot \vec{S}_{2} \vec{a}_{1} \cdot \vec{n}+2 \vec{S}_{1} \cdot \dot{\vec{S}}_{2} \vec{v}_{1} \cdot \vec{n}+\dot{\vec{S}}_{1} \cdot \vec{S}_{2} \vec{v}_{1} \cdot \vec{n}-\dot{\vec{S}}_{1} \cdot \vec{S}_{2} \vec{v}_{2} \cdot \vec{n}\right. \\
& \left.-2 \dot{\vec{S}}_{2} \cdot \vec{n} \vec{S}_{1} \cdot \vec{v}_{1}-\vec{S}_{1} \cdot \vec{n} \vec{S}_{2} \cdot \vec{a}_{1}-\dot{\vec{S}}_{1} \cdot \vec{n} \vec{S}_{2} \cdot \vec{v}_{1}\right) \\
& -3 \vec{S}_{1} \cdot \vec{v}_{2} \times \vec{n} \vec{S}_{1} \cdot \vec{S}_{2} \vec{a}_{1} \cdot \vec{n} \\
& +\vec{S}_{2} \cdot \vec{v}_{2} \times \vec{n}\left(\frac{3}{2}\left(\dot{\vec{S}}_{1} \cdot \vec{v}_{2} \vec{S}_{1} \cdot \vec{n}+\vec{S}_{1} \cdot \vec{v}_{2} \dot{\vec{S}}_{1} \cdot \vec{n}\right)\right. \\
& \left.-3 \vec{v}_{2} \cdot \vec{n}\left(3 \dot{\vec{S}}_{1} \cdot \vec{S}_{1}-5 \dot{\vec{S}}_{1} \cdot \vec{n} \vec{S}_{1} \cdot \vec{n}\right)\right) \\
& -\frac{3}{2} \dot{\vec{S}}_{2} \cdot \vec{v}_{1} \times \vec{n}\left(S_{1}^{2} \vec{v}_{1} \cdot \vec{n}-5\left(\vec{S}_{1} \cdot \vec{n}\right)^{2} \vec{v}_{1} \cdot \vec{n}\right) \\
& +\frac{3}{2} \dot{\vec{S}}_{1} \cdot \vec{v}_{1} \times \vec{n}\left(\left(\vec{v}_{1} \cdot \vec{n}-\vec{v}_{2} \cdot \vec{n}\right) \vec{S}_{1} \cdot \vec{S}_{2}-\vec{S}_{1} \cdot \vec{n} \vec{S}_{2} \cdot \vec{v}_{1}\right) \\
& +\frac{3}{2}\left(\vec{S}_{2} \cdot \vec{a}_{2} \times \vec{n}+\dot{\vec{S}}_{2} \cdot \vec{v}_{2} \times \vec{n}\right)\left(-S_{1}^{2} \vec{v}_{1} \cdot \vec{n}+2 \vec{S}_{1} \cdot \vec{v}_{1} \vec{S}_{1} \cdot \vec{n}-5\left(\vec{S}_{1} \cdot \vec{n}\right)^{2} \vec{v}_{1} \cdot \vec{n}\right) \\
& +\frac{3}{2} \vec{S}_{1} \cdot \vec{a}_{1} \times \vec{n}\left(-\vec{S}_{1} \cdot \vec{n} \vec{S}_{2} \cdot \vec{v}_{1}+\vec{S}_{1} \cdot \vec{S}_{2} \vec{v}_{1} \cdot \vec{n}\right), \\
& L_{(7)}=\vec{S}_{1} \cdot \vec{S}_{2} \times \vec{n} \dot{\vec{S}}_{1} \cdot \vec{n}+\dot{\vec{S}}_{1} \cdot \vec{S}_{2} \times \vec{n} \vec{S}_{1} \cdot \vec{n}, \\
& L_{(8)}=-4 \vec{S}_{1} \cdot \vec{S}_{2} \times \vec{n} \dot{\vec{S}}_{1} \cdot \vec{n}-13 \vec{S}_{1} \cdot \dot{\vec{S}}_{2} \times \vec{n} \vec{S}_{1} \cdot \vec{n}-4 \dot{\vec{S}}_{1} \cdot \vec{S}_{2} \times \vec{n} \vec{S}_{1} \cdot \vec{n}, \\
& L_{(9)}=\vec{S}_{1} \cdot \dot{\vec{S}}_{2} \times \vec{v}_{2} \dot{\vec{S}}_{1} \cdot \vec{n}+\dot{\vec{S}}_{1} \cdot \dot{\vec{S}}_{2} \times \vec{v}_{2} \vec{S}_{1} \cdot \vec{n}+\vec{S}_{1} \cdot \dot{\vec{S}}_{2} \times \vec{a}_{1} \vec{S}_{1} \cdot \vec{n} \\
& +\vec{S}_{1} \cdot \vec{S}_{2} \times \vec{a}_{2} \dot{\vec{S}}_{1} \cdot \vec{n}+\dot{\vec{S}}_{1} \cdot \vec{S}_{2} \times \vec{a}_{2} \vec{S}_{1} \cdot \vec{n}-\frac{1}{2} \vec{S}_{1} \cdot \vec{v}_{2} \times \vec{n} \ddot{\vec{S}}_{1} \cdot \vec{S}_{2} \\
& -\frac{1}{2} \dot{\vec{S}}_{1} \cdot \vec{v}_{1} \times \vec{n} \vec{S}_{1} \cdot \dot{\vec{S}}_{2}-\frac{1}{2} \ddot{\vec{S}}_{1} \cdot \vec{v}_{2} \times \vec{n} \vec{S}_{1} \cdot \vec{S}_{2}-\dot{\vec{S}}_{1} \cdot \vec{v}_{2} \times \vec{n} \dot{\vec{S}}_{1} \cdot \vec{S}_{2}
\end{aligned}
$$




$$
\begin{aligned}
& -\frac{1}{2} \vec{S}_{1} \cdot \vec{v}_{1} \times \vec{n} \dot{\vec{S}}_{1} \cdot \dot{\vec{S}}_{2}-\vec{S}_{2} \cdot \vec{v}_{2} \times \vec{n}\left(\ddot{\vec{S}}_{1} \cdot \vec{S}_{1}+\dot{\vec{S}}_{1} \cdot \dot{\vec{S}}_{1}\right) \\
& -3\left(\vec{S}_{2} \cdot \vec{a}_{2} \times \vec{n}+\dot{\vec{S}}_{2} \cdot \vec{v}_{2} \times \vec{n}\right)\left(\dot{\vec{S}}_{1} \cdot \vec{S}_{1}-\dot{\vec{S}}_{1} \cdot \vec{n} \vec{S}_{1} \cdot \vec{n}\right) \\
& -3 \dot{\vec{S}}_{2} \cdot \vec{v}_{1} \times \vec{n} \dot{\vec{S}}_{1} \cdot \vec{n} \vec{S}_{1} \cdot \vec{n}-\frac{1}{2} \dot{\vec{S}}_{2} \cdot \vec{a}_{1} \times \vec{n}\left(3\left(\vec{S}_{1} \cdot \vec{n}\right)^{2}+S_{1}^{2}\right) \\
& -\frac{3}{2} \vec{S}_{1} \cdot \vec{S}_{2} \times \vec{n} \ddot{\vec{S}}_{1} \cdot \vec{n} \vec{v}_{2} \cdot \vec{n}+\frac{3}{2} \vec{S}_{1} \cdot \dot{\vec{S}}_{2} \times \vec{n} \dot{\vec{S}}_{1} \cdot \vec{n} \vec{v}_{1} \cdot \vec{n} \\
& -3 \dot{\vec{S}}_{1} \cdot \vec{S}_{2} \times \vec{n} \dot{\vec{S}}_{1} \cdot \vec{n} \vec{v}_{2} \cdot \vec{n}-\frac{3}{2} \ddot{\vec{S}}_{1} \cdot \vec{S}_{2} \times \vec{n} \vec{S}_{1} \cdot \vec{n} \vec{v}_{2} \cdot \vec{n} \\
& +\frac{3}{2} \dot{\vec{S}}_{1} \cdot \dot{\vec{S}}_{2} \times \vec{n} \vec{S}_{1} \cdot \vec{n} \vec{v}_{1} \cdot \vec{n}, \\
L_{(10)}= & -\frac{1}{2} \vec{S}_{1} \cdot \dot{\vec{S}}_{2} \times \vec{n} \ddot{\vec{S}}_{1} \cdot \vec{n}-\dot{\vec{S}}_{1} \cdot \dot{\vec{S}}_{2} \times \vec{n} \dot{\vec{S}}_{1} \cdot \vec{n}-\frac{1}{2} \ddot{\vec{S}}_{1} \cdot \dot{\vec{S}}_{2} \times \vec{n} \vec{S}_{1} \cdot \vec{n},
\end{aligned}
$$

and also:

$$
\begin{aligned}
L_{\mathrm{S}_{1}^{3}}^{\mathrm{NLO}}= & C_{1\left(E S^{2}\right)} \frac{G^{2} m_{2}}{r^{5} m_{1}} L_{[1]}+C_{1\left(E S^{2}\right)} \frac{G^{2} m_{2}^{2}}{r^{5} m_{1}^{2}} L_{[2]}+C_{1\left(B S^{3}\right)} \frac{G m_{2}}{r^{4} m_{1}^{2}} L_{[3]} \\
& +C_{1\left(B S^{3}\right)} \frac{G^{2} m_{2}}{r^{5} m_{1}} L_{[4]}+C_{1\left(B S^{3}\right)} \frac{G^{2} m_{2}^{2}}{r^{5} m_{1}^{2}} L_{[5]}+C_{1\left(E S^{2}\right)} \frac{G m_{2}}{r^{3} m_{1}^{2}} L_{[6]} \\
& +C_{1\left(E S^{2}\right)} \frac{G^{2} m_{2}}{r^{4} m_{1}} L_{[7]}+C_{1\left(B S^{3}\right)} \frac{G m_{2}}{r^{3} m_{1}^{2}} L_{[8]}+C_{1\left(B S^{3}\right)} \frac{G m_{2}}{r^{2} m_{1}^{2}} L_{[9]},
\end{aligned}
$$

with the pieces:

$$
\begin{aligned}
L_{[1]}= & \frac{1}{2}\left(-\vec{S}_{1} \cdot \vec{v}_{1} \times \vec{n}+\vec{S}_{1} \cdot \vec{v}_{2} \times \vec{n}\right)\left(S_{1}^{2}-9\left(\vec{S}_{1} \cdot \vec{n}\right)^{2}\right) \\
L_{[2]}= & 3\left(\vec{S}_{1} \cdot \vec{v}_{1} \times \vec{n}-\vec{S}_{1} \cdot \vec{v}_{2} \times \vec{n}\right)\left(S_{1}^{2}-2\left(\vec{S}_{1} \cdot \vec{n}\right)^{2}\right) \\
L_{[3]}= & \vec{S}_{1} \cdot \vec{v}_{1} \times \vec{v}_{2} \vec{S}_{1} \cdot \vec{v}_{2} \vec{S}_{1} \cdot \vec{n} \\
& +\vec{S}_{1} \cdot \vec{v}_{1} \times \vec{n}\left(\frac{1}{2} S_{1}^{2}\left(v_{1}^{2}-2 \vec{v}_{1} \cdot \vec{v}_{2}+2 v_{2}^{2}-5 \vec{v}_{1} \cdot \vec{n} \vec{v}_{2} \cdot \vec{n}\right)\right. \\
& +\vec{S}_{1} \cdot \vec{v}_{1}\left(-\vec{S}_{1} \cdot \vec{v}_{1}+\vec{S}_{1} \cdot \vec{v}_{2}+\vec{S}_{1} \cdot \vec{n}\left(5 \vec{v}_{1} \cdot \vec{n}-6 \vec{v}_{2} \cdot \vec{n}\right)\right)-5 \vec{S}_{1} \cdot \vec{v}_{2} \vec{S}_{1} \cdot \vec{n} \vec{v}_{1} \cdot \vec{n} \\
& \left.-\frac{5}{2}\left(\vec{S}_{1} \cdot \vec{n}\right)^{2}\left(v_{1}^{2}-2 \vec{v}_{1} \cdot \vec{v}_{2}+2 v_{2}^{2}-7 \vec{v}_{1} \cdot \vec{n} \vec{v}_{2} \cdot \vec{n}\right)\right) \\
& +\vec{S}_{1} \cdot \vec{v}_{2} \times \vec{n}\left(-\frac{1}{2} S_{1}^{2}\left(v_{2}^{2}-5 \vec{v}_{1} \cdot \vec{n} \vec{v}_{2} \cdot \vec{n}\right)+\frac{5}{2}\left(\vec{S}_{1} \cdot \vec{n}\right)^{2}\left(v_{2}^{2}-7 \vec{v}_{1} \cdot \vec{n} \vec{v}_{2} \cdot \vec{n}\right)\right. \\
& \left.+\vec{S}_{1} \cdot \vec{v}_{1}\left(\vec{S}_{1} \cdot \vec{v}_{1}-\vec{S}_{1} \cdot \vec{v}_{2}-\vec{S}_{1} \cdot \vec{n}\left(4 \vec{v}_{1} \cdot \vec{n}-5 \vec{v}_{2} \cdot \vec{n}\right)\right)+5 \vec{S}_{1} \cdot \vec{v}_{2} \vec{S}_{1} \cdot \vec{n} \vec{v}_{1} \cdot \vec{n}\right) \\
& +\vec{v}_{1} \cdot \vec{v}_{2} \times \vec{n}\left(-\left(\vec{S}_{1} \cdot \vec{n}\right)^{2}\left(\vec{S}_{1} \cdot \vec{v}_{1}+\frac{5}{2} \vec{S}_{1} \cdot \vec{v}_{2}\right)+\frac{1}{2} S_{1}^{2} \vec{S}_{1} \cdot \vec{v}_{2}\right) \\
\left.L_{[4]}\right) & \frac{1}{2}\left(\vec{S}_{1} \cdot \vec{v}_{1} \times \vec{n}-\vec{S}_{1} \cdot \vec{v}_{2} \times \vec{n}\right)\left(S_{1}^{2}-5\left(\vec{S}_{1} \cdot \vec{n}\right)^{2}\right) \\
L_{[5]}= & -4\left(\vec{S}_{1} \cdot \vec{v}_{1} \times \vec{n}-\vec{S}_{1} \cdot \vec{v}_{2} \times \vec{n}\right)\left(S_{1}^{2}-5\left(\vec{S}_{1} \cdot \vec{n}\right)^{2}\right) \\
L_{[6]}= & 3\left[\left(\vec{S}_{1} \cdot \vec{v}_{1} \times \vec{a}_{1}-\vec{S}_{1} \cdot \vec{v}_{2} \times \vec{a}_{1}\right)\left(S_{1}^{2}-2\left(\vec{S}_{1} \cdot \vec{n}\right)^{2}\right)\right.
\end{aligned}
$$




$$
\begin{aligned}
& \left.+\vec{S}_{1} \cdot \vec{a}_{1} \times \vec{n}\left(S_{1}^{2}\left(\vec{v}_{1} \cdot \vec{n}-\vec{v}_{2} \cdot \vec{n}\right)-2 \vec{S}_{1} \cdot \vec{n}\left(\vec{S}_{1} \cdot \vec{v}_{1}-\vec{S}_{1} \cdot \vec{v}_{2}\right)\right)\right] \\
& -\frac{3}{2}\left[\dot{\vec{S}}_{1} \cdot \vec{S}_{1} \times \vec{v}_{1}\left(\vec{S}_{1} \cdot \vec{v}_{1}-\vec{S}_{1} \cdot \vec{v}_{2}-\vec{S}_{1} \cdot \vec{n}\left(\vec{v}_{1} \cdot \vec{n}-\vec{v}_{2} \cdot \vec{n}\right)\right)\right. \\
& -\dot{\vec{S}}_{1} \cdot \vec{v}_{1} \times \vec{v}_{2}\left(S_{1}^{2}-2\left(\vec{S}_{1} \cdot \vec{n}\right)^{2}\right) \\
& \left.-\dot{\vec{S}}_{1} \cdot \vec{v}_{1} \times \vec{n}\left(S_{1}^{2}\left(\vec{v}_{1} \cdot \vec{n}-\vec{v}_{2} \cdot \vec{n}\right)-2 \vec{S}_{1} \cdot \vec{n}\left(\vec{S}_{1} \cdot \vec{v}_{1}-\vec{S}_{1} \cdot \vec{v}_{2}\right)\right)\right], \\
L_{[7]}= & -3 \dot{\vec{S}}_{1} \cdot \vec{S}_{1} \times \vec{n} \vec{S}_{1} \cdot \vec{n}, \\
L_{[8]}= & \frac{1}{6}\left(2 \vec{S}_{1} \cdot \vec{v}_{1} \times \vec{v}_{2}\left(\dot{\vec{S}}_{1} \cdot \vec{S}_{1}-3 \dot{\vec{S}}_{1} \cdot \vec{n} \vec{S}_{1} \cdot \vec{n}\right)+\dot{\vec{S}}_{1} \cdot \vec{v}_{1} \times \vec{v}_{2}\left(S_{1}^{2}-3\left(\vec{S}_{1} \cdot \vec{n}\right)^{2}\right)\right. \\
& +\vec{S}_{1} \cdot \vec{a}_{1} \times \vec{v}_{2}\left(S_{1}^{2}-3\left(\vec{S}_{1} \cdot \vec{n}\right)^{2}\right)+\vec{S}_{1} \cdot \vec{v}_{1} \times \vec{a}_{2}\left(S_{1}^{2}-3\left(\vec{S}_{1} \cdot \vec{n}\right)^{2}\right) \\
& -6 \vec{S}_{1} \cdot \vec{v}_{1} \times \vec{n}\left(\vec{S}_{1} \cdot \vec{v}_{1} \dot{\vec{S}}_{1} \cdot \vec{n}+\dot{\vec{S}}_{1} \cdot \vec{v}_{1} \vec{S}_{1} \cdot \vec{n}+\vec{S}_{1} \cdot \vec{a}_{1} \vec{S}_{1} \cdot \vec{n}\right. \\
& \left.-\vec{v}_{2} \cdot \vec{n}\left(\dot{\vec{S}}_{1} \cdot \vec{S}_{1}-5 \dot{\vec{S}}_{1} \cdot \vec{n} \vec{S}_{1} \cdot \vec{n}\right)\right) \\
& -3 \dot{\vec{S}}_{1} \cdot \vec{v}_{1} \times \vec{n}\left(2 \vec{S}_{1} \cdot \vec{v}_{1} \vec{S}_{1} \cdot \vec{n}-\vec{v}_{2} \cdot \vec{n}\left(S_{1}^{2}-5\left(\vec{S}_{1} \cdot \vec{n}\right)^{2}\right)\right) \\
& -3 \vec{S}_{1} \cdot \vec{a}_{1} \times \vec{n}\left(2 \vec{S}_{1} \cdot \vec{v}_{1} \vec{S}_{1} \cdot \vec{n}-\vec{v}_{2} \cdot \vec{n}\left(S_{1}^{2}-5\left(\vec{S}_{1} \cdot \vec{n}\right)^{2}\right)\right) \\
& +6 \vec{S}_{1} \cdot \vec{v}_{2} \times \vec{n}\left(\vec{S}_{1} \cdot \vec{v}_{1} \dot{\vec{S}}_{1} \cdot \vec{n}+\dot{\vec{S}}_{1} \cdot \vec{v}_{1} \vec{S}_{1} \cdot \vec{n}+\vec{S}_{1} \cdot \vec{a}_{1} \vec{S}_{1} \cdot \vec{n}\right. \\
& \left.-\vec{v}_{2} \cdot \vec{n}\left(\dot{\vec{S}}_{1} \cdot \overrightarrow{S_{1}}-5 \dot{\vec{S}}_{1} \cdot \vec{n} \vec{S}_{1} \cdot \vec{n}\right)\right) \\
& +3 \dot{\vec{S}}_{1} \cdot \vec{v}_{2} \times \vec{n}\left(2 \vec{S}_{1} \cdot \vec{v}_{1} \vec{S}_{1} \cdot \vec{n}-\vec{v}_{2} \cdot \vec{n}\left(S_{1}^{2}-5\left(\vec{S}_{1} \cdot \vec{n}\right)^{2}\right)\right) \\
& \left.+3 \vec{S}_{1} \cdot \vec{a}_{2} \times \vec{n}\left(2 \vec{S}_{1} \cdot \vec{v}_{1} \vec{S}_{1} \cdot \vec{n}+\vec{v}_{1} \cdot \vec{n}\left(S_{1}^{2}-5\left(\vec{S}_{1} \cdot \vec{n}\right)^{2}\right)\right)\right), \\
L_{[9]}= & -\frac{1}{3} \vec{S}_{1} \cdot \vec{a}_{2} \times \vec{n}\left(\dot{\vec{S}}_{1} \cdot \vec{S}_{1}-3 \dot{\vec{S}} \cdot \vec{n} \vec{S}_{1} \cdot \vec{n}\right)-\frac{1}{6} \dot{\vec{S}}_{1} \cdot \vec{a}_{2} \times \vec{n}\left(S_{1}^{2}-3\left(\vec{S}_{1} \cdot \vec{n}\right) .\right.
\end{aligned}
$$

As can be seen in the result above we have grouped together terms according to their mass ratios and Wilson coefficients, and the total number/order of their higher-order time derivatives. At this stage this result is rather bulky, but it is easy to see that after the reduction of the higher-order action to an ordinary action by the removal of higher-order time derivative terms, we will only be left with such pieces as the first 4 ones in $L_{S_{1}^{2} S_{2}}^{\mathrm{NLO}}$ and the first 5 ones in $L_{S_{1}^{3}}^{\mathrm{NLO}}$, which becomes significantly more compact. The EOMs can also be derived directly from this higher-order action, and then reduced at the level of the EOMs, as was pointed out in [12].

However, before we will proceed in future work to handle via redefinitions the higherorder time derivatives appearing in the cubic-in-spin sector at this order, we will need to also take into account all the contributions to the action in this sector at this order, which originate from lower-order redefinitions of the variables made at lower-order sectors in order to remove higher-order time derivatives there, as was shown in detail in section 6 of [12]. First, for example, we recall that we have kinematic contributions as noted in eq. (5.28) of [12], that are linear in the spin, but have no field coupling. Those are required here to NLO as follows:

$$
L_{\mathrm{kin}}=-\vec{S} \cdot \vec{\Omega}-\frac{1}{2}\left(1+\frac{3}{4} v^{2}\right) \epsilon_{i j k} S_{k} v^{j} a^{i},
$$


where $S_{i j}=\epsilon_{i j k} S_{k}$, and $\Omega_{i j}=\epsilon_{i j k} \Omega_{k}$. At LO, e.g., we define the following shift of the positions, $\Delta \vec{y}_{I}$, according to

$$
\vec{y}_{1} \rightarrow \vec{y}_{1}+\frac{1}{2 m_{1}} \vec{S}_{1} \times \vec{v}_{1}
$$

and similarly for particle 2 with $1 \leftrightarrow 2$, to remove the leading accelerations. Note that as of the NLO linear-in-spin level higher-order time derivatives of spin also appear, where it was shown how to generically treat these in section 5 of [47]. Yet, since the leading spin redefinition is of higher $\mathrm{PN}$ order, terms quadratic in the leading redefinition contribute only at the next-to-NNLO (NNNLO) level. Therefore, here it is sufficient to consider the redefinition of the spins to linear order.

To recap, let us list the additional contributions coming from lower-order variable redefinitions that we will have from other sectors. From position shifts in lower-order sectors we will have:

1. The LO (1.5PN) position shift in eq. (5.24) implemented to linear order on the NLO quadratic-in-spin (spin1-spin2 + spin-squared) sectors.

2. The above LO position shift implemented to quadratic order on the Newtonian and LO spin-orbit sectors.

3. The above LO position shift to cubic order implemented on the Newtonian sector.

4. The NLO position shift at 2.5PN order in eq. (6.20) of [12] implemented to linear order on the LO quadratic-in-spin sectors.

5. The NLO position shifts at 3PN order in eqs. (6.30), (6.43) of [12] implemented to linear order on the shifted LO spin-orbit sector.

The leading redefinition of spin (of $2 \mathrm{PN}$ order) in eq. (6.21) of [12] will not contribute to our sector. From spin redefinitions, i.e. rotations of the spin, we will have then:

1. The spin redefinitions at $2.5 \mathrm{PN}$ order in eqs. (6.31), (6.44) of [12] implemented to linear order on the LO quadratic-in-spin sectors.

2. The spin redefinitions at $3 \mathrm{PN}$ order, which were required at the LO cubic-in-spin sector [11], implemented to linear order on the LO spin-orbit sector.

In a future publication we will present the full details of these redefinitions and the contributions from lower-order sectors, which add up to the reduced effective action in this sector.

\section{Conclusions}

In this work we derived for the first time the complete NLO cubic-in-spin PN effective action for the interaction of generic compact binaries via the self-contained EFT formulation for gravitating spinning objects in [12], and its extension in this work to the leading sector, where gravitational non-linearities are considered at an order in the spins that is beyond 
quadratic. This sector, which enters at the $4.5 \mathrm{PN}$ order for rapidly-rotating compact objects, completes finite-size effects up to this PN order, and is the first sector completed beyond the current state of the art for generic compact binary dynamics at the $4 \mathrm{PN}$ order. Once again the EFT of gravitating spinning objects has enabled a push in the state of the art in PN Gravity. Yet the analysis in this work indicates that going beyond this sector into the intriguing gray area of table 1 may become extremely intricate.

We have seen that at this order in spins with nonlinearities in gravity we have to take into account additional terms, which arise from a new type of worldline couplings, due to the fact that at this order the Tulczyjew gauge, which involves the linear momentum, can no longer be approximated only in terms of the four-velocity, as the latter approximation differs from the linear momentum by a spin-dependent part of an order $\mathcal{O}\left(R S^{2}\right)$. The spindependent correction gives rise to new "composite" couplings from the gauge of rotational DOFs. It is interesting to consider whether these new couplings have an insightful physical interpretation.

As we noted in section 1 one of the main motivations for us to tackle this sector was also to see what happens when we go to a sector at order higher than quadratic in the spins and nonlinear in gravity, which corresponds to a gravitational Compton scattering with quantum spins of $s \geq 3 / 2$, and to possibly also get an insight on the non-uniqueness of fixing its amplitude from factorization when spins of $s \geq 5 / 2$ are involved [36]. From [12] and the analysis in section 4 , we can see that going to an order quintic in the spins, or in the quantum case to $s=5 / 2$, exactly corresponds to where the spin-dependent correction to $p_{\mu}$ in eq. (4.2) has to be taken into account at quadratic order. We will discuss this interesting connection between the classical and the quantum levels at a future publication. A general observation that we can clearly make already is that even-parity sectors in $l$, see table 1, are easier to handle than odd ones. In the quantum context this corresponds to the greater ease of dealing with bosons compared to fermions.

Unless all the additional terms from section 4 conspire to cancel out eventually, we would obtain an effective action that differs from that with the gauge used in lower-spin sectors, involving only the four-velocity. Even still, it could be that when computing the consequent observable quantities, such as the binding energy, or the EOMs, one finds that this difference does not matter, and the two gauges are physically equivalent. In a forthcoming publication we will present the resulting Hamiltonian, EOMs, and gaugeinvariant quantities, such as the binding energy, and get an answer to these questions, including self-consistency checks of the method [52].

At the moment it is not clear whether computations carried out within a scattering amplitudes framework can capture all the classical effects derived in this paper. The generic results in this work can serve to streamline such a framework, as that which was initiated in $[39,40]$, or provide crosschecks for the conjectured result for the scattering angle at one-loop level in the restricted case of black holes with aligned spins in [43].

\section{Acknowledgments}

We thank Yu-tin Huang and Jung-Wook Kim for related discussions. We are also grateful to Roger Morales and Fei Teng for additional careful crosschecks on our results. The work 
of ML was supported by the European Research Council under the European Union's Horizon 2020 Framework Programme FP8/2014-2020 "preQFT" grant no. 639729, "Strategic Predictions for Quantum Field Theories" project, and by the European Union's Horizon 2020 research and innovation programme under the Marie Skłodowska-Curie grant agreements No. 847523 'INTERACTIONS' and No. 764850 'SAGEX'. We also acknowledge support from the Carlsberg Foundation and from the Danish National Research Foundation (DNRF91).

Open Access. This article is distributed under the terms of the Creative Commons Attribution License (CC-BY 4.0), which permits any use, distribution and reproduction in any medium, provided the original author(s) and source are credited.

\section{References}

[1] LigO Scientific, Virgo collaboration, Observation of gravitational waves from a binary black hole merger, Phys. Rev. Lett. 116 (2016) 061102 [arXiv: 1602.03837] [InSPIRE].

[2] L. Blanchet, Gravitational radiation from post-Newtonian sources and inspiralling compact binaries, Living Rev. Rel. 17 (2014) 2 [arXiv:1310.1528] [INSPIRE].

[3] A. Buonanno and T. Damour, Effective one-body approach to general relativistic two-body dynamics, Phys. Rev. D 59 (1999) 084006 [gr-qc/9811091] [InSPIRE].

[4] W.D. Goldberger and I.Z. Rothstein, An effective field theory of gravity for extended objects, Phys. Rev. D 73 (2006) 104029 [hep-th/0409156] [INSPIRE].

[5] B. Kol and M. Smolkin, Non-relativistic gravitation: from Newton to Einstein and back, Class. Quant. Grav. 25 (2008) 145011 [arXiv:0712.4116] [INSPIRE].

[6] M. Levi, Next to Leading Order gravitational Spin1-Spin2 coupling with Kaluza-Klein reduction, Phys. Rev. D 82 (2010) 064029 [arXiv:0802.1508] [INSPIRE].

[7] J.B. Gilmore and A. Ross, Effective field theory calculation of second post-Newtonian binary dynamics, Phys. Rev. D 78 (2008) 124021 [arXiv:0810.1328] [InSPIRE].

[8] M. Levi, Next to leading order gravitational spin-orbit coupling in an effective field theory approach, Phys. Rev. D 82 (2010) 104004 [arXiv: 1006.4139] [INSPIRE].

[9] S. Foffa and R. Sturani, Effective field theory calculation of conservative binary dynamics at third post-Newtonian order, Phys. Rev. D 84 (2011) 044031 [arXiv:1104.1122] [INSPIRE].

[10] M. Levi, Binary dynamics from spin1-spin2 coupling at fourth post-Newtonian order, Phys. Rev. D 85 (2012) 064043 [arXiv:1107.4322] [INSPIRE].

[11] M. Levi and J. Steinhoff, Leading order finite size effects with spins for inspiralling compact binaries, JHEP 06 (2015) 059 [arXiv:1410.2601] [INSPIRE].

[12] M. Levi and J. Steinhoff, Spinning gravitating objects in the effective field theory in the post-Newtonian scheme, JHEP 09 (2015) 219 [arXiv:1501.04956] [INSPIRE].

[13] M. Levi and J. Steinhoff, Next-to-next-to-leading order gravitational spin-orbit coupling via the effective field theory for spinning objects in the post-Newtonian scheme, JCAP 01 (2016) 011 [arXiv: 1506. 05056] [INSPIRE].

[14] M. Levi and J. Steinhoff, Next-to-next-to-leading order gravitational spin-squared potential via the effective field theory for spinning objects in the post-Newtonian scheme, JCAP 01 (2016) 008 [arXiv: 1506. 05794] [INSPIRE]. 
[15] S. Foffa, P. Mastrolia, R. Sturani and C. Sturm, Effective field theory approach to the gravitational two-body dynamics, at fourth post-Newtonian order and quintic in the Newton constant, Phys. Rev. D 95 (2017) 104009 [arXiv: 1612.00482] [INSPIRE].

[16] S. Foffa, P. Mastrolia, R. Sturani, C. Sturm and W.J. Torres Bobadilla, Static two-body potential at fifth post-Newtonian order, Phys. Rev. Lett. 122 (2019) 241605 [arXiv: 1902.10571] [INSPIRE].

[17] J. Blümlein, A. Maier and P. Marquard, Five-loop static contribution to the gravitational interaction potential of two point masses, Phys. Lett. B 800 (2020) 135100 [arXiv: 1902.11180] [INSPIRE].

[18] M. Levi, A.J. Mcleod and M. Von Hippel, $N^{3} L O$ gravitational spin-orbit coupling at order $G^{4}$, arXiv:2003.02827 [INSPIRE].

[19] M. Levi, A.J. Mcleod and M. Von Hippel, NNNLO gravitational quadratic-in-spin interactions at the quartic order in $G$, arXiv:2003.07890 [INSPIRE].

[20] M. Levi and F. Teng, NLO gravitational quartic-in-spin interaction, arXiv:2008.12280 [INSPIRE].

[21] M. Levi and J. Steinhoff, EFTofPNG: a package for high precision computation with the effective field theory of post-Newtonian gravity, Class. Quant. Grav. 34 (2017) 244001 [arXiv: 1705. 06309] [INSPIRE].

[22] M. Levi, Effective field theories of post-Newtonian gravity: a comprehensive review, Rept. Prog. Phys. 83 (2020) 075901 [arXiv:1807.01699] [InSPIRE].

[23] B.M. Barker and R.F. O'Connell, Gravitational two-body problem with arbitrary masses, spins, and quadrupole moments, Phys. Rev. D 12 (1975) 329 [INSPIRE].

[24] R.A. Porto, Post-Newtonian corrections to the motion of spinning bodies in NRGR, Phys. Rev. D 73 (2006) 104031 [gr-qc/0511061] [INSPIRE].

[25] E. Poisson, Gravitational waves from inspiraling compact binaries: the quadrupole moment term, Phys. Rev. D 57 (1998) 5287 [gr-qc/9709032] [INSPIRE].

[26] R.A. Porto and I.Z. Rothstein, Next to leading order Spin(1)Spin(1) effects in the motion of inspiralling compact binaries, Phys. Rev. D 78 (2008) 044013 [Erratum ibid. 81 (2010) 029905] [arXiv: 0804.0260] [INSPIRE].

[27] J. Steinhoff, S. Hergt and G. Schaefer, Spin-squared Hamiltonian of next-to-leading order gravitational interaction, Phys. Rev. D 78 (2008) 101503 [arXiv:0809.2200] [INSPIRE].

[28] S. Hergt and G. Schaefer, Higher-order-in-spin interaction Hamiltonians for binary black holes from Poincaré invariance, Phys. Rev. D 78 (2008) 124004 [arXiv:0809.2208] [INSPIRE].

[29] S. Hergt, J. Steinhoff and G. Schaefer, Reduced Hamiltonian for next-to-leading order spin-squared dynamics of general compact binaries, Class. Quant. Grav. 27 (2010) 135007 [arXiv: 1002.2093] [INSPIRE].

[30] S. Hergt and G. Schaefer, Higher-order-in-spin interaction Hamiltonians for binary black holes from source terms of Kerr geometry in approximate ADM coordinates, Phys. Rev. D 77 (2008) 104001 [arXiv:0712.1515] [INSPIRE].

[31] V. Vaidya, Gravitational spin Hamiltonians from the S matrix, Phys. Rev. D 91 (2015) 024017 [arXiv: 1410.5348 ] [INSPIRE].

[32] S. Marsat, Cubic order spin effects in the dynamics and gravitational wave energy flux of compact object binaries, Class. Quant. Grav. 32 (2015) 085008 [arXiv:1411.4118] [InSPIRE]. 
[33] J. Vines and J. Steinhoff, Spin-multipole effects in binary black holes and the test-body limit, Phys. Rev. D 97 (2018) 064010 [arXiv:1606.08832] [InSPIRE].

[34] Z. Bern, C. Cheung, R. Roiban, C.-H. Shen, M.P. Solon and M. Zeng, Scattering amplitudes and the conservative Hamiltonian for binary systems at third post-Minkowskian order, Phys. Rev. Lett. 122 (2019) 201603 [arXiv:1901.04424] [INSPIRE].

[35] Z. Bern, C. Cheung, R. Roiban, C.-H. Shen, M.P. Solon and M. Zeng, Black hole binary dynamics from the double copy and effective theory, JHEP 10 (2019) 206 [arXiv: 1908.01493] [INSPIRE].

[36] N. Arkani-Hamed, T.-C. Huang and Y.-t. Huang, Scattering amplitudes for all masses and spins, arXiv:1709.04891 [INSPIRE].

[37] F. Cachazo and A. Guevara, Leading singularities and classical gravitational scattering, JHEP 02 (2020) 181 [arXiv: 1705.10262] [INSPIRE].

[38] A. Guevara, Holomorphic classical limit for spin effects in gravitational and electromagnetic scattering, JHEP 04 (2019) 033 [arXiv:1706. 02314] [INSPIRE].

[39] M.-Z. Chung, Y.-T. Huang, J.-W. Kim and S. Lee, The simplest massive S-matrix: from minimal coupling to black holes, JHEP 04 (2019) 156 [arXiv:1812.08752] [INSPIRE].

[40] M.-Z. Chung, Y.-T. Huang and J.-W. Kim, Classical potential for general spinning bodies, JHEP 09 (2020) 074 [arXiv: 1908.08463] [INSPIRE].

[41] M. Levi and J. Steinhoff, Complete conservative dynamics for inspiralling compact binaries with spins at fourth post-Newtonian order, arXiv:1607.04252 [INSPIRE].

[42] B. Kol, M. Levi and M. Smolkin, Comparing space+time decompositions in the post-Newtonian limit, Class. Quant. Grav. 28 (2011) 145021 [arXiv:1011.6024] [INSPIRE].

[43] A. Guevara, A. Ochirov and J. Vines, Scattering of spinning black holes from exponentiated soft factors, JHEP 09 (2019) 056 [arXiv: 1812.06895] [INSPIRE].

[44] D. Binosi and L. Theussl, JaxoDraw: a graphical user interface for drawing Feynman diagrams, Comput. Phys. Commun. 161 (2004) 76 [hep-ph/0309015] [INSPIRE].

[45] D. Binosi, J. Collins, C. Kaufhold and L. Theussl, JaxoDraw: a graphical user interface for drawing Feynman diagrams. Version 2.0 release notes, Comput. Phys. Commun. 180 (2009) 1709 [arXiv:0811.4113] [INSPIRE].

[46] J.A.M. Vermaseren, Axodraw, Comput. Phys. Commun. 83 (1994) 45 [inSPIRE].

[47] M. Levi and J. Steinhoff, Equivalence of ADM Hamiltonian and effective field theory approaches at next-to-next-to-leading order spin1-spin2 coupling of binary inspirals, JCAP 12 (2014) 003 [arXiv: 1408.5762] [INSPIRE].

[48] W. Tulczyjew, Motion of multipole particles in general relativity theory, Acta Phys. Polon. 18 (1959) 393.

[49] W. Dixon, Dynamics of extended bodies in general relativity. I. Momentum and angular momentum, Proc. Roy. Soc. Lond. A 314 (1970) 499.

[50] R. Schattner, The uniqueness of the center-of-mass in general relativity, Gen. Rel. Grav. 10 (1979) 395 [INSPIRE].

[51] R. Schattner, The center-of-mass in general relativity, Gen. Rel. Grav. 10 (1979) 377 [INSPIRE].

[52] M. Levi and R. Morales, in preparation (2020). 